\title{
Comprehensive bioinformatics analysis of the characterization and determination underlying mechanisms of over-expression and co-expression of genes residing on $20 \mathrm{q}$ in colorectal cancer
}

\author{
Daojiang Lii ${ }^{1}$, Changwei Lin ${ }^{1}$, Miao Chen ${ }^{1}$, Nanpeng $\mathrm{Li}^{1}$, Yuheng Du ${ }^{1}$, Chen Su${ }^{1}$, \\ Chunxing Yang ${ }^{1}$, Ni Gong ${ }^{1}$, Hao $\mathrm{Wu}^{1}{ }^{1}$, Runliu $\mathrm{Wu}^{1}{ }^{1}$, Arad Jain ${ }^{3}$, Yi Zhang ${ }^{1,2}$ and \\ Xiaorong Li ${ }^{1,2}$ \\ ${ }^{1}$ Department of General Surgery, The Third Xiangya Hospital of Central South University, Changsha, Hunan 410013, China \\ ${ }^{2}$ Center for Experimental Medicine, The Third Xiangya Hospital of Central South University, Changsha, Hunan 410013, China \\ ${ }^{3}$ College of Arts and Science, University of Virginia, Charlottesville, Virginia 22904, The United States of America
}

Correspondence to: Xiaorong Li, email: lixiaorong@medmail.com.cn

Yi Zhang, email: yzhangxy3@126.com

Keywords: 20q, CNA, co-expression, colorectal cancer, adjacent gene

Received: April 14, $2017 \quad$ Accepted: July 19, $2017 \quad$ Published: August 10, 2017

Copyright: Li et al. This is an open-access article distributed under the terms of the Creative Commons Attribution License 3.0 (CC BY 3.0), which permits unrestricted use, distribution, and reproduction in any medium, provided the original author and source are credited.

\section{ABSTRACT}

The Long arm of chromosome $20(20 q)$ is closely related to the development of colorectal cancer, so identifying the expression profile of genes on $20 \mathrm{q}$ through a comprehensive overview is indispensable. In this article, preliminar experimental data, several available databases and bioinformatics tools such as the Cancer Genome Atlas, the Encyclopedia of DNA Elements, the JASPAR database and starBase were combined to analyze the correlation between genes and chromosomal aberrations, microRNA and transcription factors, as well as to explore the expression feature and potential regulative mechanism. The results showed that the most frequently unregulated genes in colorectal cancer arelocated on chromosome $20 q$, present a significant CNA-mRNA correlation.Furthermore, the genes with mRNA overexpression showed co-expression features and tended to be clustered within the same genomic neighborhoods. Then, several genes were selected to carry out further analysis and demonstrated that shared transcription factors, a conserved bidirectional promoter, and competition for a limited pool of microRNAin the 3'UTR of mRNA may be the underlying mechanisms behind the co-expression of physically adjacent genes.Finally, the databases, Lentivirus ShRNA, and qPCR were used to find that these adjacent genes with co-expression cooperatively participated in the same biological pathways associated with the pathogenesis and development of colorectal cancer.

\section{INTRODUCTION}

Chromosomal aberrations are presented in about $85 \%$ of colorectal cancer (CRC) patients and occur as nonrandom events, generally involving losses in $8 \mathrm{p}, 17 \mathrm{p}$ and $18 \mathrm{q}$, and gain in 7p, 7q, 8q, 13q and 20q [1]. Among these, gains of $20 \mathrm{q}$ were first found through banding analysis in colorectal cancer [2] and it had been observed $>65 \%$ of CRCs [3], which suggests that the genes encoded on $20 \mathrm{q}$ have a key role in contributing to the phenotype of CRC when overexpressed. Further in-depth research demonstrated that the amplification of $20 \mathrm{q}$ was conspicuously complex, not all genes with copy number alterations (CNA or CNV) will alter gene expression, and the synergistic effect of multiple genes was important in tumor development $[4,5]$, thus it become necessary to explore the relationship between CNA and alteration of gene dosage, as well as the comprehensive expression characteristics of the genes on $20 \mathrm{q}$. 
Now,compared to pure experimentation with gene function studies, which focuses on one or several selected genes and biological pathways, bioinformatics and database resources have become an important means to analyze, compare and interpret data from a global scale and macroscopic level [6]. The vast amount of molecular data involving genomes, transcriptomes, proteomes and resources from other biological layers are publicly available [6, 7], So in this study, several available databases, bioinformatics tools, preliminarexperimental data were reasonably combined and employed to analyze the correlation between genes located on $20 \mathrm{q}$, CNA, microRNA, transcription, and so forth. Through this comprehensive comparison method, we were able to explore the expression characteristics and potential regulative mechanisms of these genes, through which we can better understand the molecular basis of how these genes on $20 \mathrm{q}$ were involved in CRC, and identify novel genomic targets for therapeutic intervention.

\section{RESULTS}

\section{The genes located on chromosome 20q were frequently upregulated in colorectal cancer}

Once the somatic mutations of all genes in colon adenocarcinoma were analyzed by COSMIC, the result showed that the majority of top 20 genes with somatic mutations are mRNA over expression (18/20) and CNA gain, except 2 genes (PCLO with high methylation and APC with high point mutation). When the somatic mutations was only restricted to "Gene expression", we found that the top 20 genes mainly presented mRNA overexpression and CNA gain, and all were located on 20q, as shown through NCBI and Ensemble identification (Supplementary Figure 1). Further analysis through Map Viewer showed that these genes distributed on 3 adjacent DNA fragments, including 20q13.33, 20q13.32, 20q13.31, 20q13.13, 20q13.12, 20q11.23, 20q11.22 and 20q11.21 (Figure 1A and Supplementary Figure 1). Oncomine was used to confirm gene expression, as compared to normal tissue.TCGA and GEO data all demonstrated that these genes have higher expression in colorectal samples (Figure 1B/1C). Then we used The Human Protein Atlas to clarify the protein expression in colorectal cancer. tThe results revealed that protein showed different cytoplasmic or nuclear immunoreactivity. Proteins such as TM9SF4 and POFUT1 have strong positivity but CTNBL1 has a weak to moderate immunoreactivity and PIGU presented negatively (Figure 1D), which is in line with the fact that mRNA level increases do not translate into increased abundance of the corresponding proteins [8]. Finally, we selected 3 genes with high expression mRNA and protein to carry out survival analysis and found these genes didn't decrease the survival rate of colorectal patients (Figure 1E).

\section{The genes located on chromosome $20 \mathrm{q}$ that are frequently unregulated in colorectal cancer showed significant CNA-mRNA correlation}

As mentioned above, we found that the top 20 genes with frequently unregulated in colorectal cancer all resided on the long arm of chromosome 20 (20q), In particular these genes with mRNA over expression were accompanied by CNA gain, which remind us DNA amplification may be the reason primary for gene mRNA overexpression. To illustrate this, as showed in Figure 2A, we first selected 8 unregulated genes located on 20q13.33 (YYHDF1), 20q13.32 (NELFCD or TH1L), 20q13.13 (STAU1), 20q13.12 (ZWIM3), 20q11.23 (AAR2 or C20orf4), 20q11.22 (PIGU) and 20q11.21 (PLAGL2 and POFUT1) for an additional somatic mutations analysis in CRC through cBioPortal (Colorectal Adenocarcinoma (TCGA, Provisional) 633 samples). The results showed that Amplification and mRNA overexpression are the main mutations of 8 genes (Figure 2B). Then we downloaded the TCGA data with quantifiable CNA and mRNA measurements for CNA-mRNA correlation analysis, in which the 8 genes showed significant CNA-mRNA correlation $(\mathrm{p}<0.0001, \mathrm{r}>0.65)$ (Figure $3 \mathrm{C})$.

\section{The genes with mRNA overexpression on 20q exhibited significant co-expression and tended to be clustered within the same genomic neighborhoods}

Interestingly, further analysis of 3 genes (PLAGL2, POFUT1and STAU1) located on chromosome $20 \mathrm{q}$ and frequently unregulated in colorectal cancer exhibited significant co-expression levels (PLAGL2 vs. POFUT1, $\mathrm{r}=0.84, \mathrm{p}<0.0001$; POFUT1 vs.STAU1, $\mathrm{r}=0.66, \quad \mathrm{p}<0.0001)$, which prompted us to explore the co-expression of all genes located on 20q (Figure 3A). 2 genes,STAU1 and POFUT1,which are ranked 2 and 5 respectively in all genes with mRNA overexpression in CRC (Supplementary Figure 1 and Figure 1A),were selected for this study. For the convenience of calculations and comparable statistic results (Figure 3B), the co-expression data between all genes (20436) and STAU1 and POFUT1 in CRC was downloaded from The cBioPortal for Cancer Genomics (Colorectal Adenocarcinoma (TCGA, Provisional) 633 samples) (Supplementary Table 2). Firstly, the data revealed that there are 86 genes correlated significantly with POFUT1 (pearson Score $\geq 0.6$ ), 96.5\% (83/86). These genes are uniformly distributed on $20 \mathrm{q}$ with more genes located on 20q11.22, 20q11.21 and 20q11.23 (23\%, $13 \%$ and $19 \%$, respectively). (calculated through "gene with pearson Score $\geq 0.6$ "/"all genes located on this DNA region") (Figure 3C and Supplementary Table 2). It is noteworthy that 20q11.22, 20q11.21 and 
A

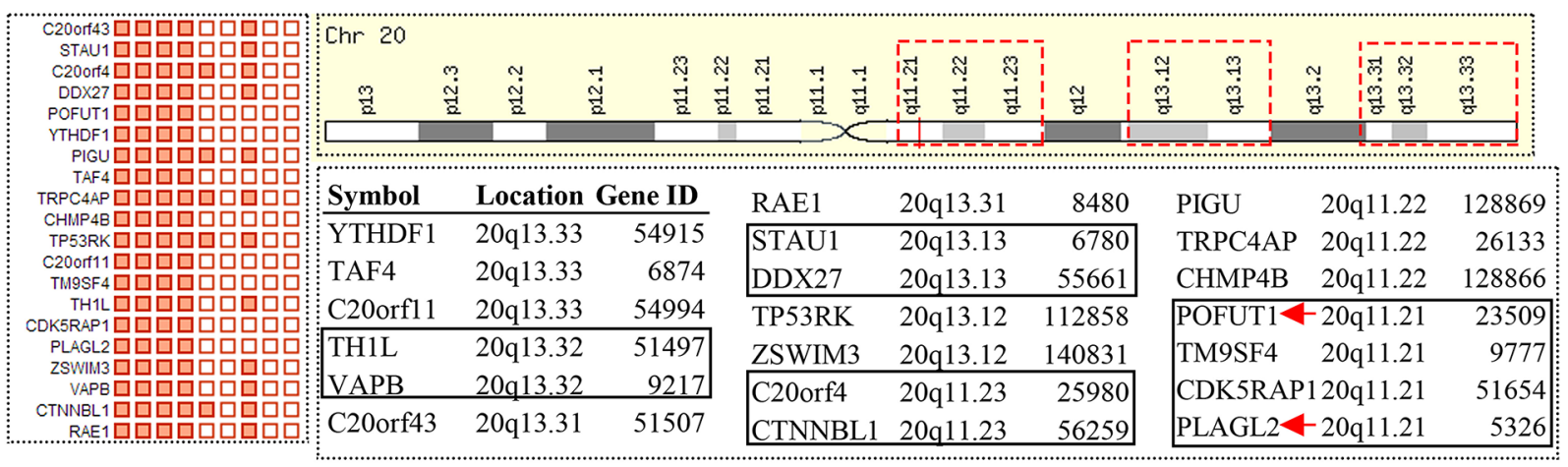

B

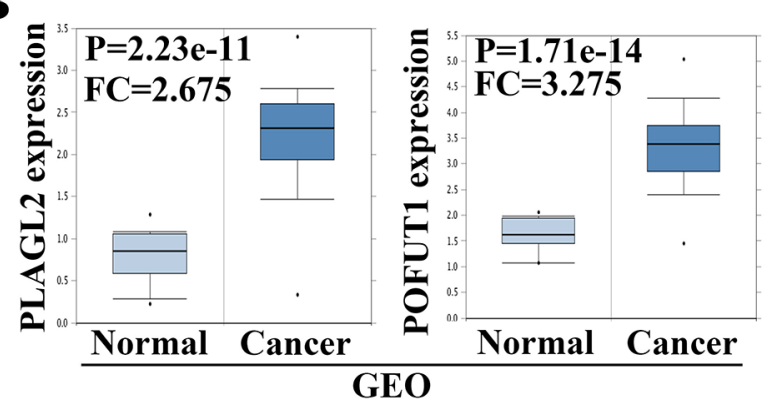

D
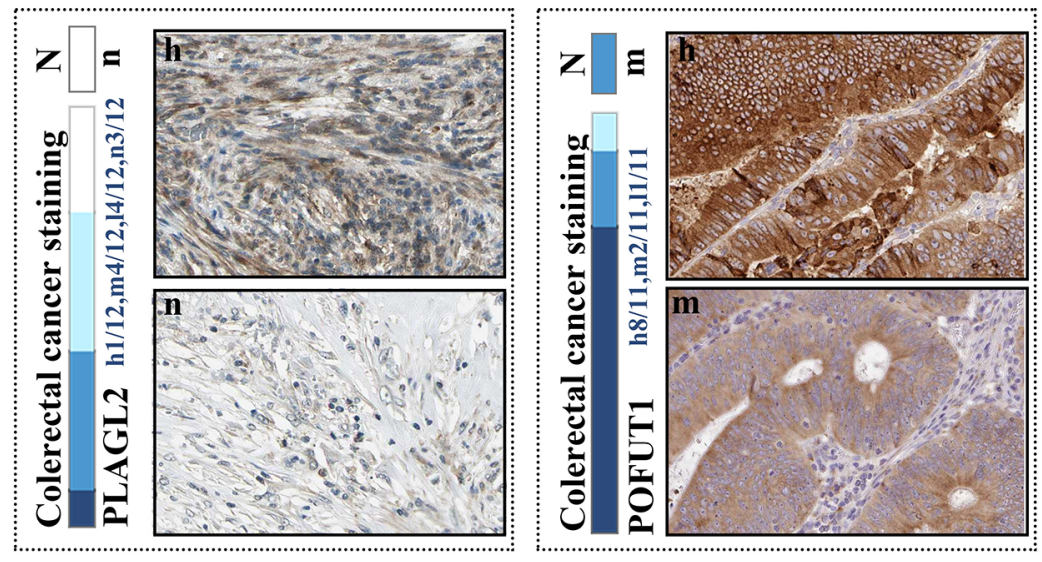

C
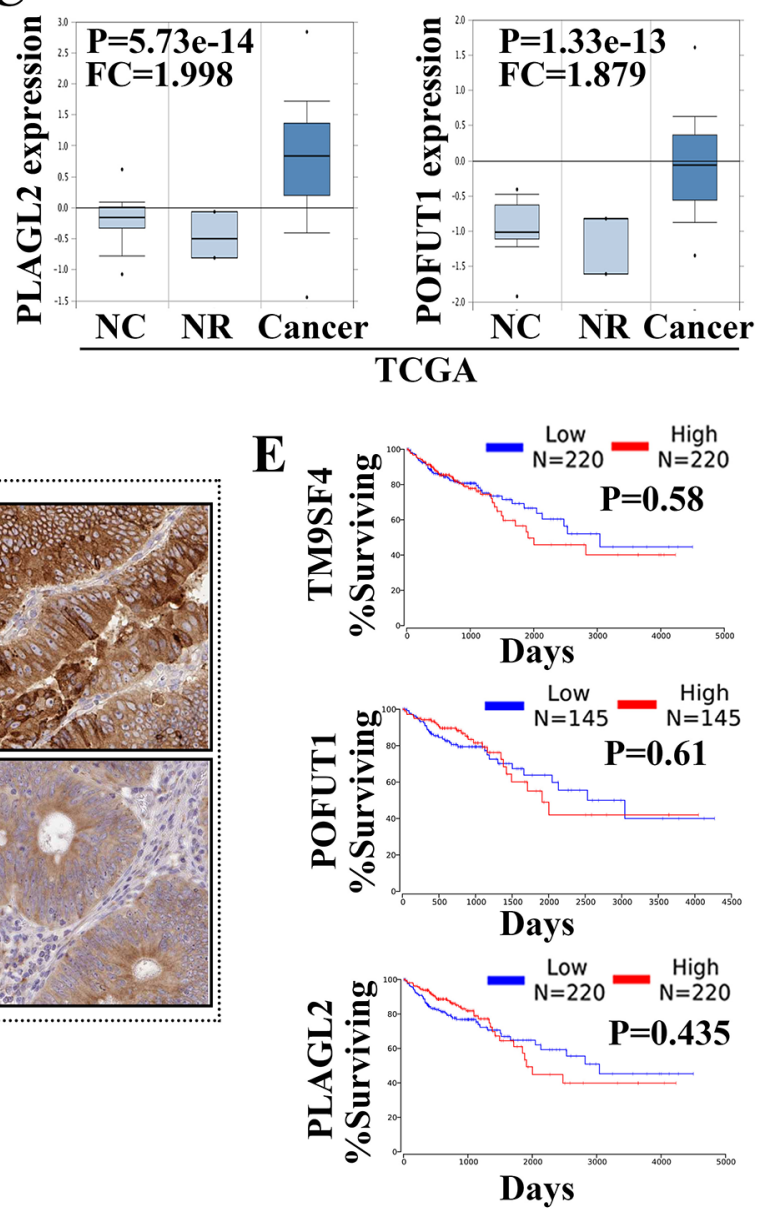

Figure 1: The genes located on chromosome 20q were frequently unregulated in colorectal cancer. (A) The top 20 genes with mRNA over expression in colon adenocarcinoma (COSMIC study Id: COSU376), the matrix on the left side of the figure list the top unregulated 20 genes in colon cancer (more details can be found in Supplementary Figure 1); the distribution of 20 genes on 20q and the symbol, location and gene ID of each gene were showed on the right side of the figure, red dashed box show all genes located in 3 DNA fragments, black rectangle was used to distinguish the genes located in 20q13.33, 20q13.32, 20q13.31, 20q13.13, 20q13.12, 20q11.23, 20q11.22, 20q11.21. (B/C) The representative gene (PLAGL2 and POFUT1) expression in colorectal cancer and normal tissues, which analyzed by Oncomine(GSE9348 and TCGA Colorectal), NC: normal colon, NR: normal rectum, FC: fold change. All other genes demonstrated same result as PLAGL2 (data not show, more detail can be found in Ocomine). (D) The representative protein expression derived from The Human Protein Atlas (PLAGL2 and POFUT1 with moderate to strong immunoreactivity was show here, data derived from antibody-based protein profiling using immunohistochemistry), N: normal tissue, n: negative,h: strong or high. (E) Survival analysis of PLAGL2, POFUT1 and TM9SF4 in colorectal cancer, data derived from TCGA. 
20q11.23 and POFUT1 (20q11.21) are within genomic neighborhoods. We then wanted to explore whether the genes correlated significantly with POFUT1 and STAU1 also tend to lie adjacent to each other in the genome. 50 genes with same CNA and located adjacent to each other in the 20q11.21 (POFUT1) and 20q13.13 (STAU1) were selected for study, as demonstrated in Figure 3C/3D and Supplementary Table 2. 34 genes on 20q11.21 and 17 genes on 20q13.13 remained for analysis and the results found that the genes correlated significantly with POFUT1 and STAU1 have a distinct disjunctive distribution and are clustered into co-regulated groups

A

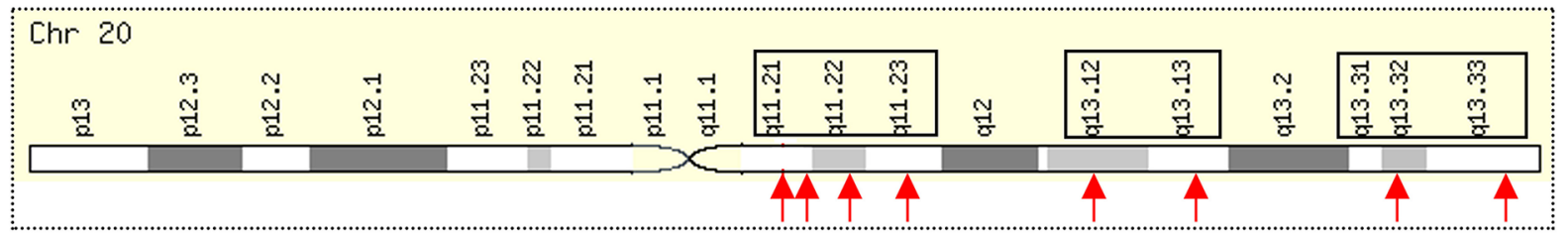

B

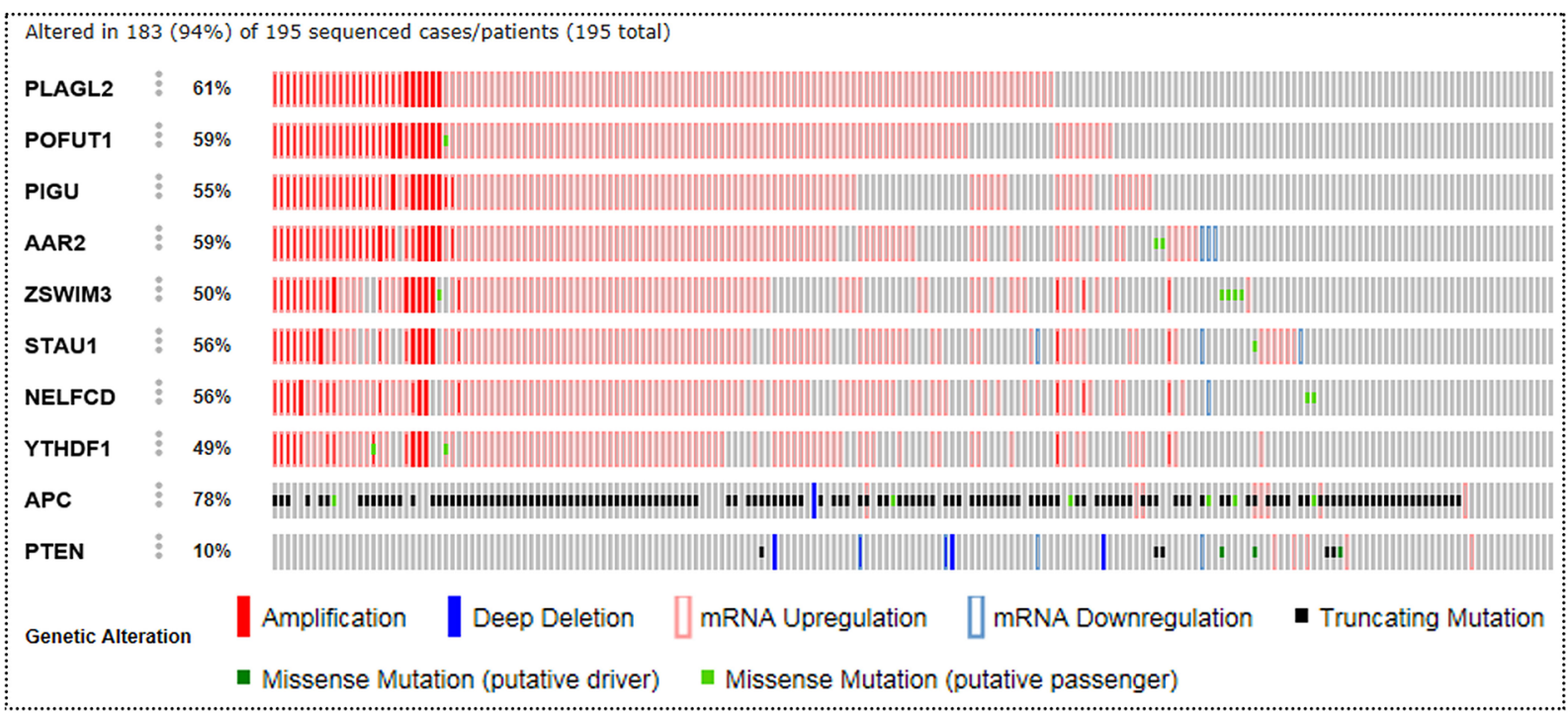

C

20q11.21

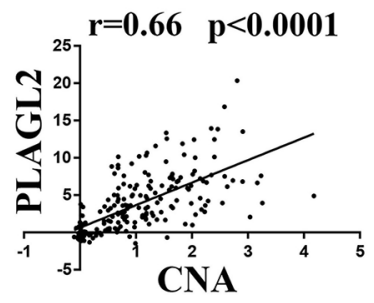

20q13.12

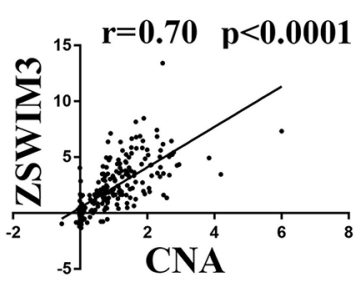

20q11.21

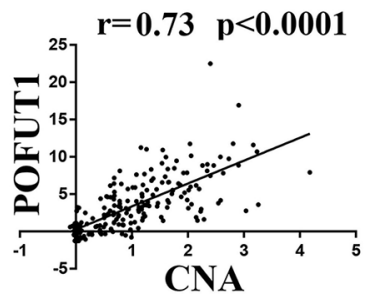

20q13.13

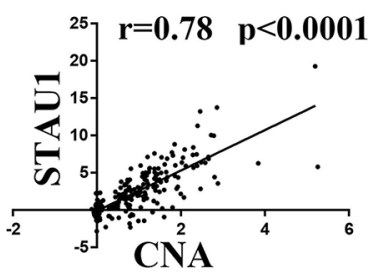

20q11.22

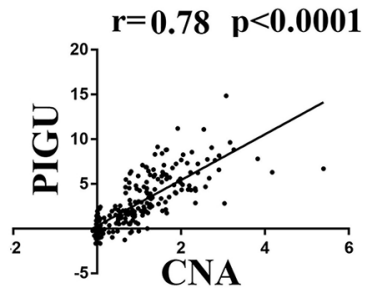

20q13.32

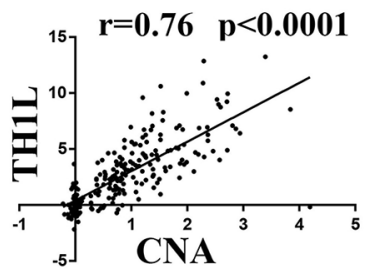

20q11.23

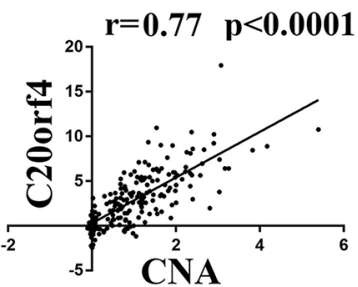

20q13.33

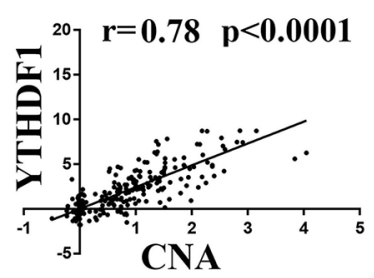

Figure 2: 8 genes located on chromosome 20q and frequently unregulated in colorectal cancer showed significant CNA-mRNA correlation. (A) 8 unregulated genes located on 20q13.33 (YYHDF1), 20q13.32 (NELFCD or TH1L), 20q13.13 (STAU1), 20q13.12 (ZWIM3), 20q11.23 (AAR2 or C20orf4), 20q11.22 (PIGU) and 20q11.21 (PLAGL2 and POFUT1) were selected for somatic mutations analysis in CRC. (B) Amplification and mRNA up regulation are the main mutations of 8 genes; the result is evaluated by "The cBioPortal for Cancer Genomics database (bowel-colorectal adenocarcinoma)". (C) 8 genes showed significant CNA-mRNA correlation $(\mathrm{p}<0.0001, \mathrm{r}>0.65)$. 
A

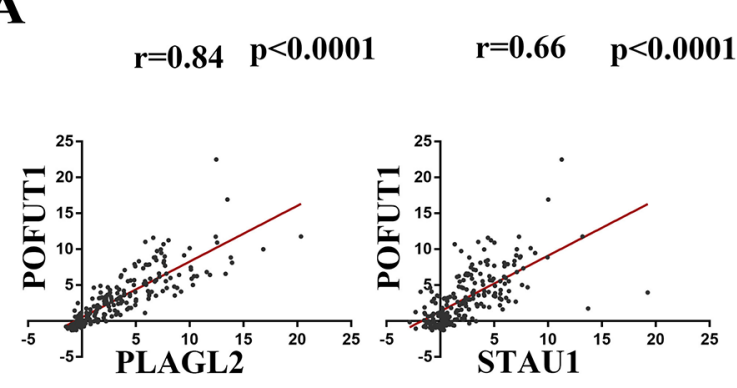

B

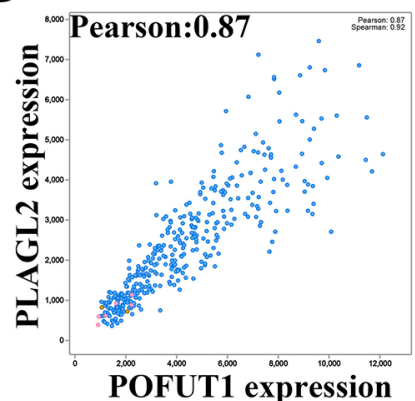

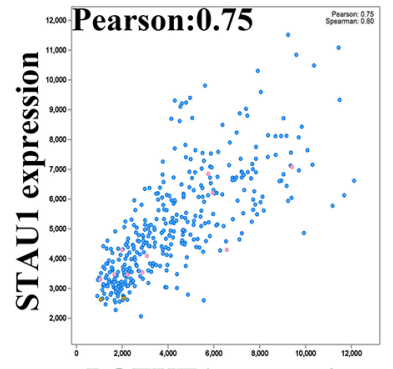

POFUT1 expression
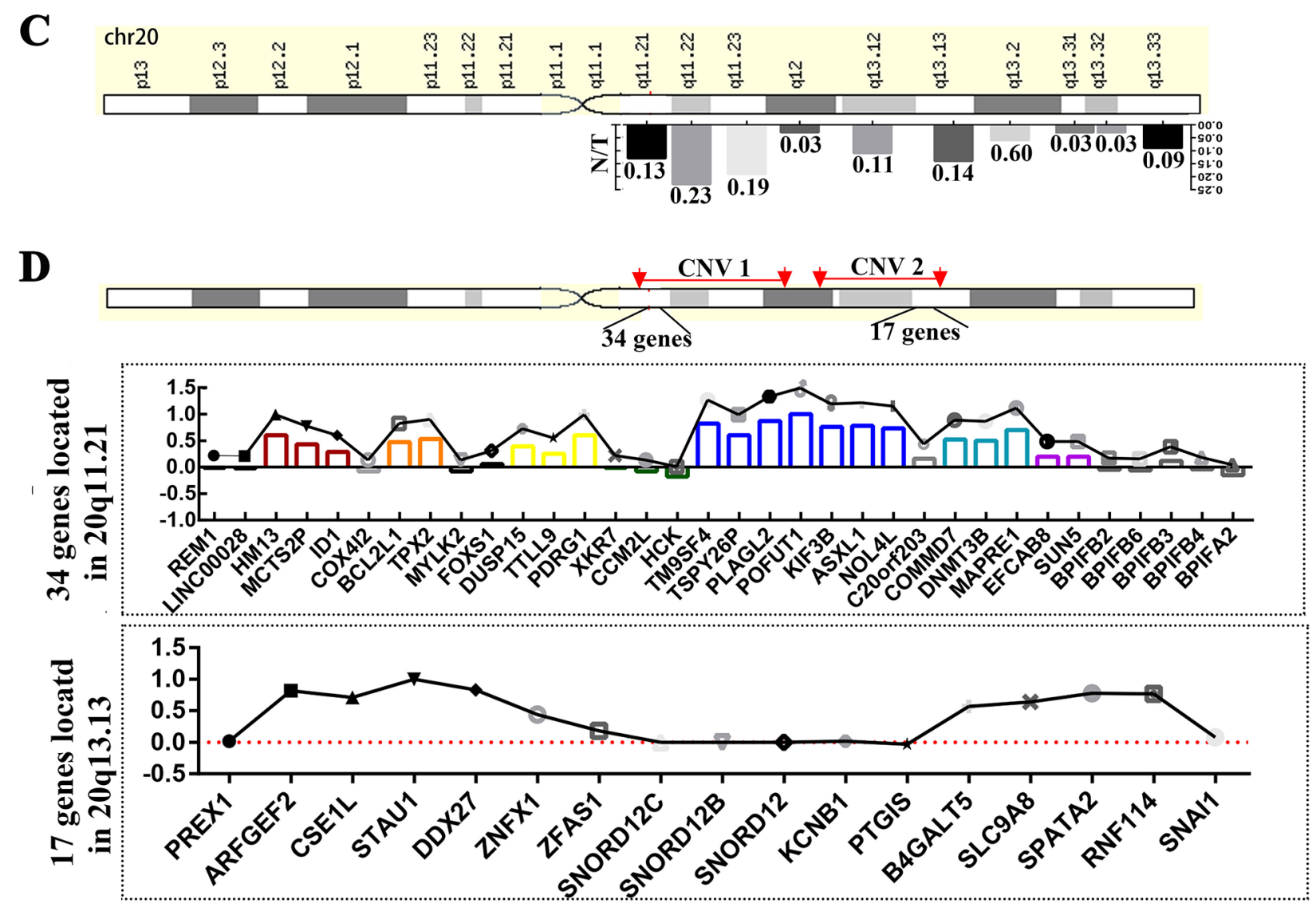

Figure 3: The genes located on chromosome 20q with similar expression levels tend to be clustered within the same genomic neighborhoods. (A) The mRNA expression of POFUT1 and PLAGL2 or STAU1 shows extremely obvious positive correlation, 218 TCGA sample data with quantifiable mRNA measurements (Supplementary Table 1) were used for mRNA-mRNA evaluation. (B) The co-expression data between all genes (20436 genes) and STAU1 and POFUT1 in CRC was download from the cBioPortal for Cancer Genomics (Colorectal Adenocarcinoma, 633 samples) and show same obvious positive correlation between POFUT1 and PLAGL2 or STAU1. (C) 86 genes correlated significantly with POFUT1 (Pearson score $\geq 0.6), 96.5 \%$ (83/86) gene distributed on 20q and more genes located on $20 \mathrm{q} 11.22,20 \mathrm{q} 11.21$ and $20 \mathrm{q} 11.23(23 \%, 13 \%$ and $19 \%$, respectively, calculated through gene with Pearson score $\geq 0.6 /$ all genes located on this DNA region). (D) 34 genes adjacent with POFUT1 and 17 genes adjacent with STAU2 presented a distinct discontinuous distribution and are clustered into co-regulated groups of 1-7 genes such as POFUT1 co-expressed with its adjacent genes TM9SF4(0.82), PLAGL2(0.87), KIF3B(0.76) and ASXL1(0.78), STAU1 co-expressed with ARFGEF2(0.82),CSE1L(0.71) and DDX27(0.83) (gene cluster presented with different color); the ordinate means Pearson score(genes vs. POFUT1 and POFUT1 was set to 1 in the above diagram, genes vs. STAU1 and STAU1 was set to 1 in the image below), CNV1 and CNV2 means two groups of genes located on same focal amplification fragment. (More detail can be found in Supplementary Table 2) 
of 2-7 genes.For example, POFUT1 co-expressed with its adjacent genes TM9SF4(0.82), PLAGL2(0.87), KIF3B(0.76) and ASXL1(0.78), STAU1 co-expressed with ARFGEF2(0.82), CSE1L(0.71) and DDX27(0.83). In addition, interaction networks predicted by GeneMANIA also support this conclusion (Figure 4A).

\section{Shared transcription factors in promoter sequences may be one mechanism contributing to co-expression of adjacent genes}

Previous studies have discovered that a large proportion of transcriptome in human is linearly arranged in small groups of adjacent genes which tend to be regulated by the same transcription factors [9], so the adjacent gene group including TM9SF4, POFUT1, PLAGL2, KIF3B and ASXL1 was arranged for further study. Venn Diagrams indicated that the promoters of these 5 genes share 60 transcription factors which was supported by ChIP-Seq evidence (Figure 4B,
Supplementary Table 3). In addition, the co-expression analysis between these shared transcription factors and POFUT1 was carried out using co-expression data derived from cBioPortal, The results showed $55 \% \quad(33 / 60)$ presented Pearson Score $\geq 0$ such as TGIF2(0.64), myc(0.43) and SP3(0.32); 45\%(27/60) presented Pearson Score $\leq 0$ such as $\operatorname{RCOR} 1(-0.38)$, SOX13(-0.37) and SP1(-0.19) (Supplementary Table $3)$,indicating that which indicated POFUT1 can be activated or repressed by the transcription factors as discussed above. Then we performed gene set enrichment analysis by means of Enrichr software. Two gene-set libraries about the transcription category were used in this article ( PWMs from TRANSFAC and JASPAR, The ENCODE transcription factor gene-set). The enrichment analysis results for the ENCODE gene-set library found that 2-4 adjacent genes may share same the transcription factor, such as ASXL1,KIF3B, POFUT1 and PLAGL2, which shareEGR1 $(\mathrm{p}<0.0001)$ (Figure 4C and Supplementary Table 3), However analysis of

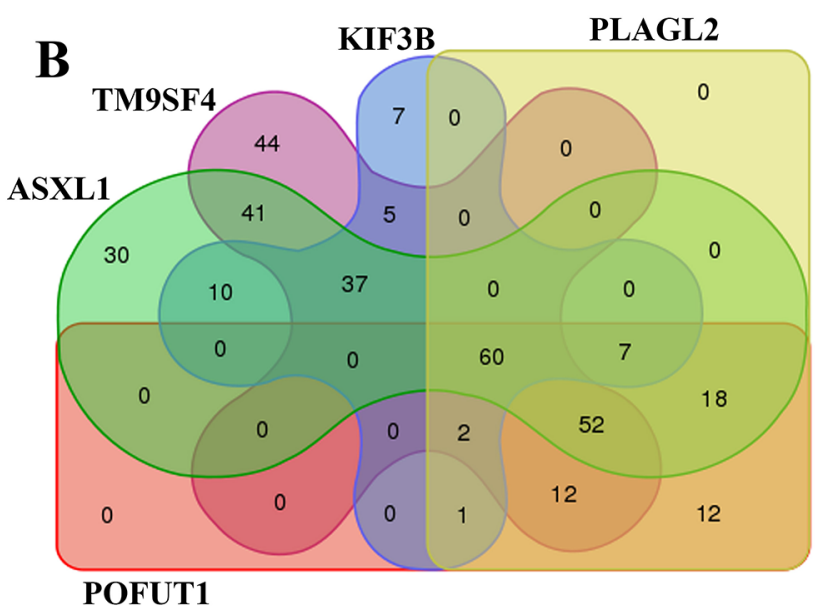

D

\begin{tabular}{|c|c|c|}
\hline \multicolumn{3}{|c|}{ ENCODE TF ChIP-seq 2015} \\
\hline Term & P-value & Genes \\
\hline EGR1_Hl-hESC_hg19 & 0.000 & ASXL1;KIF3B;POFUT1;PLAGL2 \\
\hline EP300_MEL cell line_mm9 & 0.000 & ASXL1;KIF3B;POFUT1;PLAGL2 \\
\hline ETS1_MEL cell line_mm9 & 0.000 & ASXL1;KIF3B;POFUT1;PLAGL2 \\
\hline KAT2A_GM12878_hg19 & 0.000 & ASXL1;POFUT1;PLAGL2;TM9SF4 \\
\hline NR3C1_HepG2_hg19 & 0.001 & ASXL1;TM9SF4 \\
\hline RAD21_HepG2_hg19 & 0.001 & ASXL1;KIF3B;POFUT1;PLAGL2 \\
\hline RELA_GM12892_hg19 & 0.002 & ASXL1;POFUT1;PLAGL2 \\
\hline MYC_HeLa-S3_hg19 & 0.002 & ASXL1;POFUT1;PLAGL2;TM9SF4 \\
\hline
\end{tabular}

\begin{tabular}{|} 
TRANSFAC and JASPAR PWMs \\
\begin{tabular}{|c|c|c|}
\hline Term & P-value & Genes \\
\hline MIB2 & 0.004 & POFUT1;PLAGL2;TM9SF4 \\
\hline SMAD4 & 0.004 & ASXL1;POFUT1;PLAGL2 \\
\hline FOXF1 & 0.040 & ASXL1;KIF3B \\
\hline TP63 & 0.045 & ASXL1;KIF3B \\
\hline REPIN1 & 0.046 & POFUT1;PLAGL2 \\
\hline UBTF & 0.048 & ASXL1;POFUT1 \\
\hline NR5A2 & 0.051 & KIF3B;PLAGL2;TM9SF4 \\
\hline EGR1 & 0.055 & POFUT1;PLAGL2 \\
\hline Stat3 & 0.065 & POFUT1;PLAGL2 \\
\hline
\end{tabular}
\end{tabular}

Figure 4: Adjacent genes with co-expression may be regulated in part by common transcription factors. (A) Interaction networks predicted by GeneMANIA also identified TM9SF4, POFUT1, PLAGL2, KIF3B and ASXL1 showed co-expression. (B) Venn diagrams indicated that the promoters of these 5 genes share 60 transcription factors supported by ChIP-Seq evidence (the transcription factors of each gene and intersections can be found in Supplementary Table 3). (C) The representative enrichment analysis results for ENCODE geneset library found that 2-4 adjacent genes may share same transcription factor (more details can be found in Supplementary Table 3). (D) The representative enrichment analysis results for PWMs from TRANSFAC and JASPAR gene-set library found that 2-4 adjacent genes may share same transcription factor (more details can be found in Supplementary Table 3). C and D analyzed by software Enrichr. 
PWMs from TRANSFAC and JASPAR gene-set libraria revealed that 2-3 genes may share the same transcription factors, such as ASXL1,POFUT1 and PLAGL2 which share SMAD4 $(p=0.004)$ (Figure 4D and Supplementary Table 3).

\section{Gene pair regulated by evolutionarily conserved bidirectional promoter may be a classic mechanism explaining co-expressions of physically adjacent genes}

As analyzed above, what is particularly intriguing in enrichment analysis of transcription factor is that gene pair, PLAGL2 and POFUT1, was the shortest physical sequence distance $(<150 \mathrm{bp})$ and are the most common 2 genes shared transcription factor. More importantly, the mRNA expression of PLAGL2 correlated significantly with that of $\operatorname{POFUT} 1(\mathrm{r}=0.87, \mathrm{p}<0.0001$, Figure $3 \mathrm{~A})$ and Venn Diagrams in Figure 4B showed PLAGL2 and POFUT1 shared all 164 transcription factors based on ChIP-Seq evidence (Supplementary Table 3). Thus, the PLAGL2 and POFUT1 pair was selected as a candidate for further study. Firstly, we employed UCSC genome browser to analyze the genomic organization. Interesting, the results suggest the human PLAGL2 and POFUT1are located on $20 \mathrm{q} 11.21$ in a head-to-head orientation, with the start of both genes are separated less than 150bp and overlapped with a putative $\mathrm{CpG}$ island. According to ENCODE v24 annotation, the intergenic region displayed high DNase 1 hypersensitivity and was enriched with hallmarks associated with transcription like H3K4me3, H3K4me1 and H3K27Ac. Furthermore, the region lacks TATA box, is highly evolutionarily conserved (Supplementary Table 4), and contains multiple potential DNA motifs including SP1,TFAP2C, E2F4, EGR1 and Ets transcription families, which have been verified to be overrepresented in the bidirectional promoter [10] (Figure 5A/5B). These result strongly indicatethat PLAGL2 and POFUT1 are a classic bidirectional gene pair. Next, as shown in Figure 6A, 89 bp nucleotide sequences, which regard as optimal bidirectional promoter by computer-assisted analyses, was retained for more thorough analysis. The results of this demonstrated that this sequence was highly evolutionarily conserved and contains multiple transcription factor binding sites, including SP1, SP3, SP2, E2F4, E2F6, EGR1 and TFAP2C (Figure 6B and Supplementary Figure 2). Finally, 244 TCGA sample data sets with quantifiable mRNA measurements (Supplementary Table 4) were used for mRNA (transcription factor)-mRNA correlation evaluation. The results showed that E2F4 and SP3 correlated significantly with POFUT1 and PLAGL2 in colon and rectal cancer (Figure 6C), which indicated E2F4 and SP3 are the most likely transcription factor binding to this bidirectional promoter and causing coexpression of the gene pair.

\section{Competition for a limited pool of microRNAs mediates cross-regulation of mRANs may be another mechanism contributing to the co- expression of adjacent genes}

Apart from the regulation of transcription in nucleus, a number of recent studies unraveled that coexpression of mRNA may be caused by the competing endogenous RNA (ceRNA) mechanism in cytoplasm. The 3'UTR of different mRNA transcripts containing common microRNA recognition elements(MRES) can compete for common microRNAs, by which different mRNA transcripts can keep co-expressing [11]. So in this article, we explore the possibility that co-expression of adjacent genes can be brought out by a ceRNA mechanism. To start with, the adjacent gene group including TM9SF4, POFUT1, PLAGL2, KIF3B and ASXL1 was arranged for this study. Venn Diagrams demonstrated that these five genes shared 9 miRNAs predicted by TargetScan (Figure 7A).Then, considering adjacent gene pairs may share most micRNAs than beyond 3 genes, POFUT1 and PLAGL2 wereselected for further study. The intersections of experimentally validated microRNAs of both genes are 12 and 11 in TarBase v7.0 number of and miRTARBASE6.0. In addition, we found the number of intersections of microRNAs in Starbase v2.0, which was processed from five miRNA prediction software programs (TargetScan, PicTar, PITA, miRanda and RNA22) and overlapped with CLIP-Seq data, is 38. As showed in Figure 7B, Figure 7C and Supplementary Table 5, PLAGL2 and POFUT1 share common 52 microRNAs as shown through experimental evidence. Furthermore these microRNAs can directlay target PLAGL2 and POFUT1 and repress the expression of both genes in colon and rectal cancer. All these results and ceRNA prediction by Starbase v2.0 (Figure 7D) suggested that the adjacent genes with co-expressionmay be mediated by ceRNA.

\section{Co-expression of adjacent genes cooperatively participate in the same biological pathways associated with the pathogenesis and development of colorectal cancer}

As discussed above, a paires of adjacent genes with co-expression were selected for further analysis which showed a sharing of transcription factors, an evolutionary conserved bidirectional promoter and/or competition for a limited pool of microRNAs may be the underlying mechanism leading to co-expression, but this can only explain a part of the phenomenon. More research needs to be done to clarify the biological process. Compared with the difficulty in interpreting the co-expression mechanism, what was really noticeable was that the biological pathways that these genes are involved in are very similar. 6 adjacent genes with co-expression characterization as shown in Figure 3(TM9SF4, PLAGL2, POFUT1, KIF3B, 
ASXL1, NOL4L) were chosen for gene-set pathway enrichment analysis. We first carried out KEGG pathway enrichment analysis for each of the 6 genes. The geneset of each gene (Pearson CorrelationCoefficient $\geq 0.2$, Supplementary Table 8) was compiled from cBioPortal for Cancer Genomics (Colorectal Adenocarcinoma (TCGA, Nature 2012) 276 samples), then a Venn analysis was performed to calculate the statistically significant intersection(s) of KEGG pathways of each gene. As shown in Table 1, 6 adjacent genes share common biological pathway such as "Endometrial cancer","Hippo signaling pathway","Choline metabolism in cancer","Pathways in cancer", "Signaling pathways regulating pluripotency of stem cells","Wnt signaling pathway", and"Adherens junction". Among these, TM9SF4, POFUT1 and KIF3B are all involved in the pathway of "Colorectal cancer". All indicated that co-expression of adjacent genes cooperatively participated in the same biological pathways associated with the pathogenesis and development of human cancers, including colorectal cancer; furthermore

A

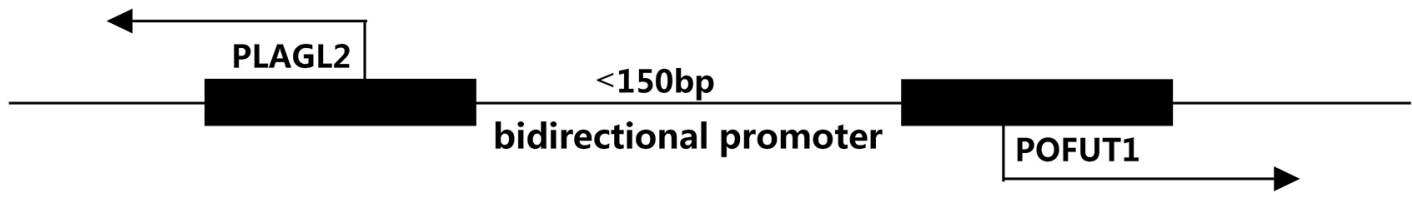

B

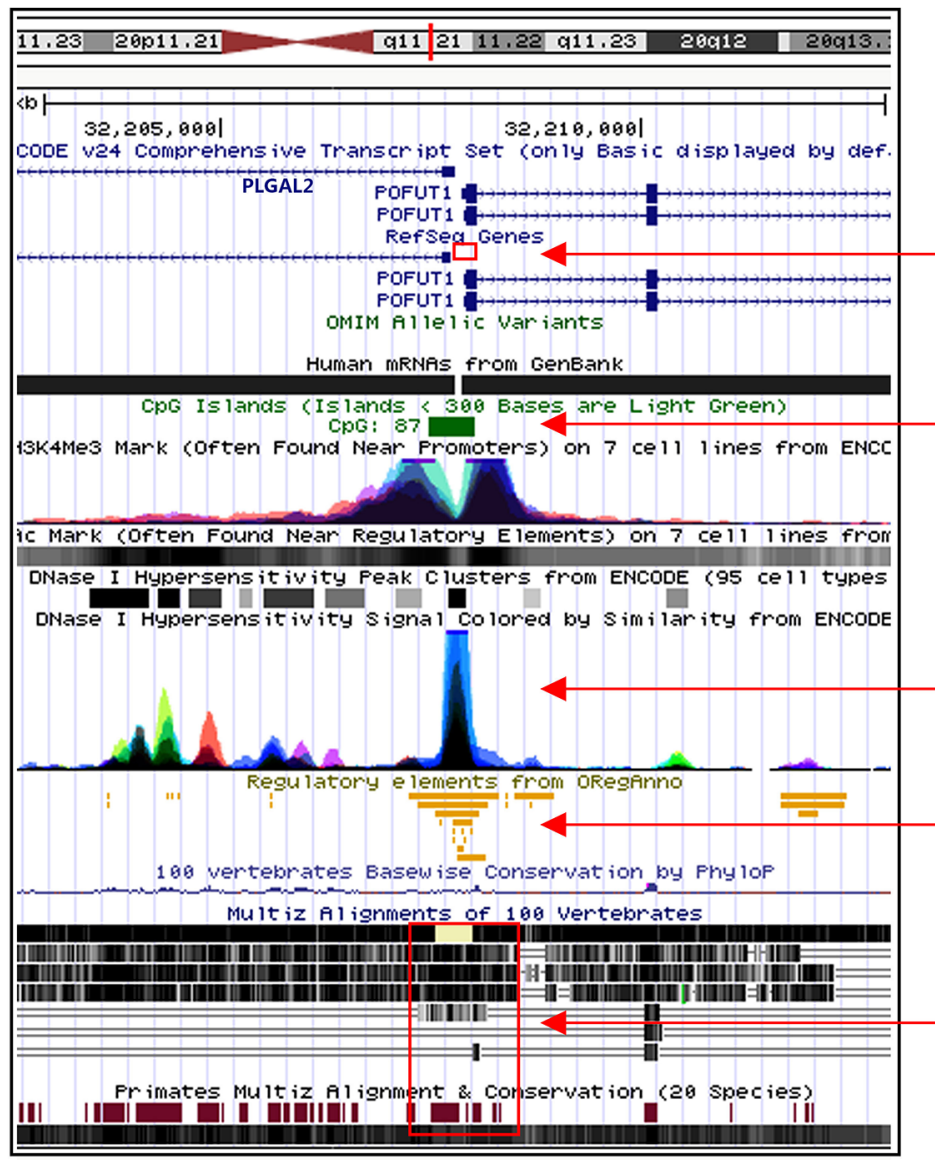

POFUT1 and PLAGL2 arranged head-to-head on opposite strands of DNA and with $<300 \mathrm{bp}$ of one another

the intergenic region between two genes overlapped with a putative $\mathrm{CpG}$ island $(87)$

the intergenic region are enriched with hallmark associated with transcription like H3K 4me3, H3K4me1 and H3K27Ac

the intergenic region displayed high DNase I hypersensitivity

POFUT1 and PLAGL2 shared same transcription factor binding sites such as SP1, TFAP2C,E2F4, EGR1 and Ets transcription factor family member

the intergenic region is highly evolutionarily conserved

Figure 5: PLAGL2 and POFUT1 are classic bidirectional gene pair. (A) Schematic representation of a bidirectional promoter between PLAGL2 and POFUT1 genes, black arrows denote transcription direction of both genes. (B) We employed UCSC genome browser(alter tracks displayed) to analyze the genomic organization, the human PLAGL2 and POFUT1 are located on 20q11.21 in a headto-head orientation, the start of both genes are separated less than $150 \mathrm{bp}$ and overlapped with a putative CpG island (87bp), according to ENCODE v24 annotation determined by a ChIP-seq assay, the intergenic region displayed high DNase 1 hypersensitivity and was enriched with histone hallmark associated with transcription like H3K4me3, H3K4me1 and H3K27Ac, furthermore the nucleotide sequence highly evolutionarily conserved (more details can be found in Supplementary Table 4) and contains multiple potential DNA motifs including SP1,TFAP2C, E2F4, EGR1 and Ets transcription family, which have been verified overrepresented in bidirectional promoter. 
A

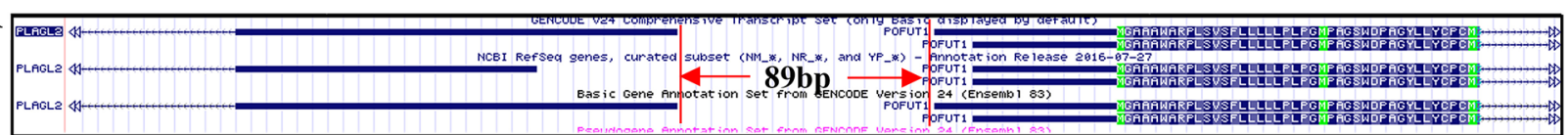

A

UCSC: the first exon of GENCODE V24,RefSeq and Ensembl are different

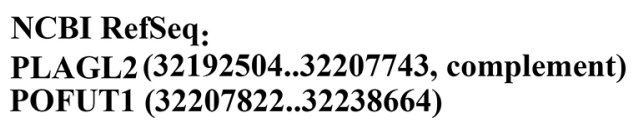

NCBI RefSeq:

PLAGL2 (32192504..32207743, complement)

POFUT1 (32207822..32238664)
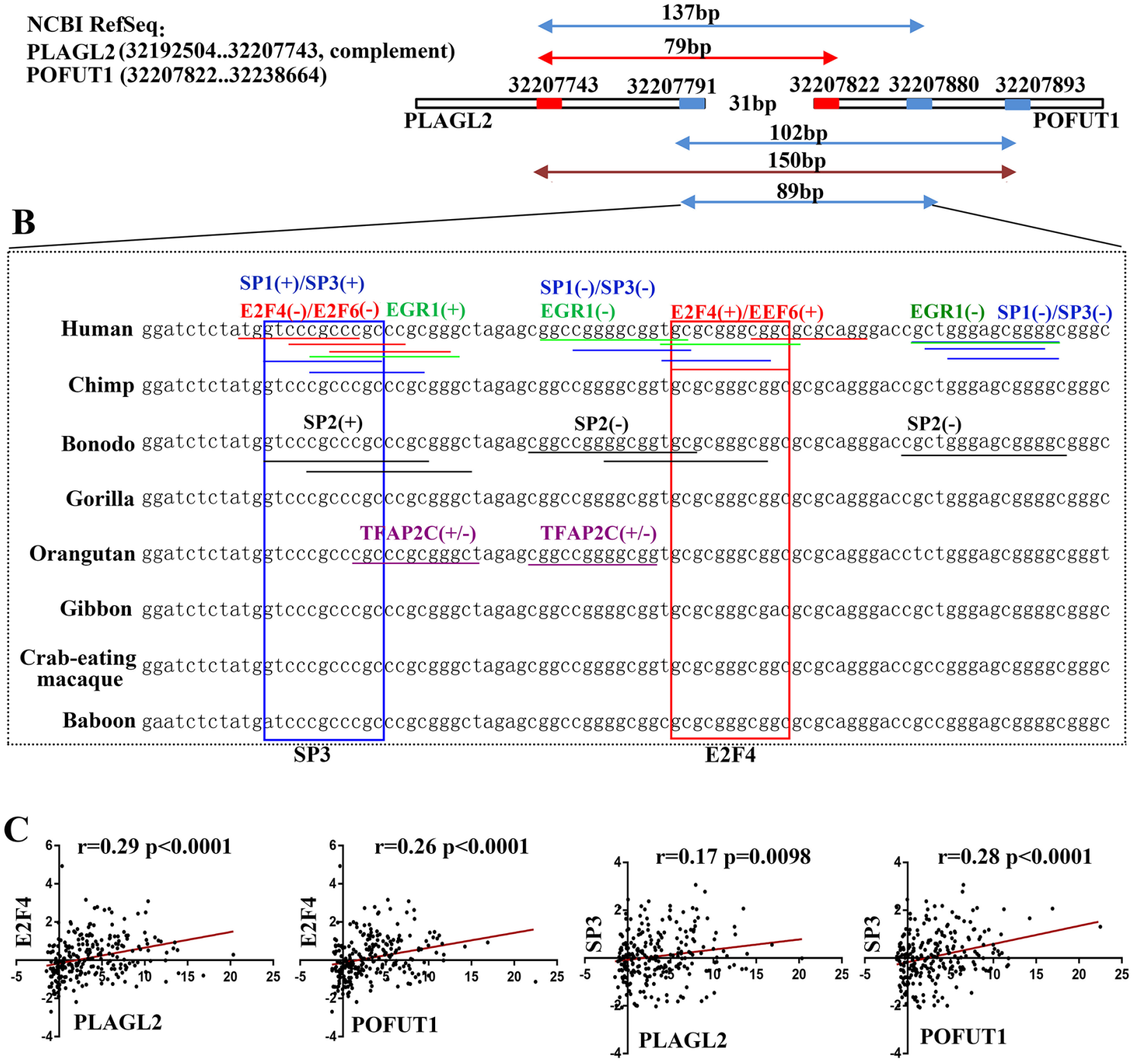

Figure 6: The optimal bidirectional promoter and transcription factor. (A) Because 3 gene database including NIBI RefSeq, ENCODE v24 and Ensembl genome browser 87 displayed different positionfor PLAGL2 and POFUT1, so we draw a schematic diagramto find out the optimal nucleotide sequence ( $89 \mathrm{bp}$ between32207791 to 32207880) for bidirectional promoter, two-way arrow denote the base number between the different start of both genes (the start derived from NIBI RefSeq was marked red and show here, and more descriptions can be found in Supplementary Table 4). (B) Base pair alignment between human and several other species in the region between 32207791 to 32207880 (89bp), the predicted transcriptions factor binding sites are marked by horizontal line with different color (SP1 and SP3 with blue, SP2 with black, E2F4 and E2F6 with red, EGR1with green, TFAP2C with purple). Two features have been observed, first and foremost, the base sequence including transcription factor binding sites are highly conserved during evolution, besides all predicted transcription factor binding sites mainly focused on 3 regions, especially TFAP2C can better binding to positive-sense $(+)$ and negativesense(-) strand. Among these transcriptions factor, E2F4 and SP3 were selected for co-expression analysis and labeled with rectangular box. (More conserved nucleotide sequence and details can be found in Supplementary Table 4 and transcriptions factor binding sites analyzed by The JASPAR database can be found in Supplementary Figure 2.) (C) Transcriptions factor E2F4 and SP3 correlated significantly with POFUT1 and PLAGL2 in colon and rectal cancer. 
these adjacent genes maintain co-expression through various mechanisms, are may be designed to participate in the common function. In addition, PLAGL2 and POFUT1, which are separated by shortest physical sequence distance $(<150 \mathrm{bp})$ and exhibit co-expression characteristics in 14 cancer types (Supplementary Table 7) were used for further analysis. 63 genes(Stable7) which can maintain correlation with POFUT1and PLAGL2 in a variety of human tumor tissues were selected for protein-protein interactions by STRING v10.5, as shown in Figure 8A. The oncogenes, $S R C$ is the most important intersection point, so we inhibited the expression of PLAGL2 and POFUT1 in SW620 cells. The results found that lowexpression of $S R C$ can be found in two knockout cells (Figure 8B), which indicate PLAGL2 and POFUT1 cooperatively participate in SRC biological pathways,
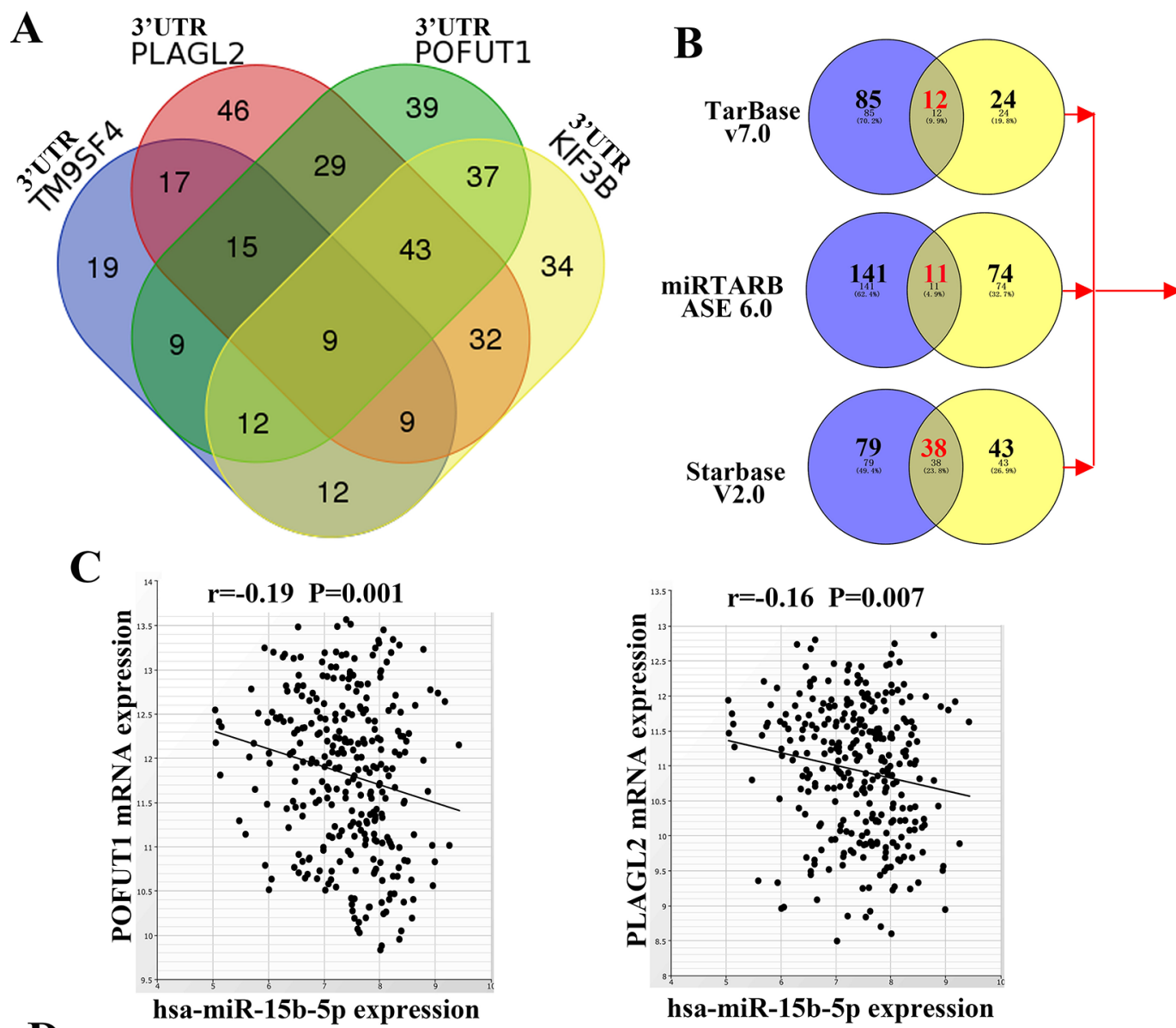

D

hsa-miR-15b-5p expression

\begin{tabular}{|c|c|c|c|c|}
\hline 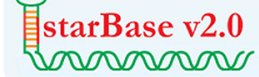 & \multicolumn{4}{|c|}{ CLIP-Seq, Pan-Cancer. } \\
\hline geneName & geneType & cernaName & cernaType & pvalue \\
\hline$\underline{\text { PLAGL2 }}$ & protein_coding & CELSR3 & protein_coding & 0.0263245 \\
\hline PLAGL2 PLAGL2 & protein_coding & \multicolumn{2}{|c|}{ POFUT1 POFUT1 protein_coding } & 0.0439591 \\
\hline$\underline{\text { PLAGL2 }}$ & protein_coding & ATXN7L3 & protein_coding & 0.00766176 \\
\hline$\underline{\text { PLAGL2 }}$ & protein_coding & FAM53C & protein_coding & 0.00924709 \\
\hline
\end{tabular}

Figure 7: Adjacent genes with co-expression, especially adjacent gene pair, may be mediated by ceRNA. (A) Venn Diagrams demonstrated that 3'UTR of five genes shared 9 miRNAs predicted by TargetScan. (B) The intersections of experimentally validated microRNAs of PLAGL2 and POFUT1 are 12 and 11 in TarBase v7.0 and miRTARBASE6.0, and the intersections of microRNAs in Starbase v2.0 is 38, the union of 3 intersections is 52, which indicated both genes share common 52 microRNAs with experimental evidence. (C) The representative anti-correlation (pearson correlation: $r<0$, p-value $<0.05$ ) between miRNA and target genes across colon and rectal cancer. (D) Starbase v2.0 predicted that POFUT1 is a perfect ceRNA of PLAGL2. (More details about Figure 7 can be found in Supplementary Table 5.) 
Table 1: The Venn result of KEGG pathway of 6 genes with co-expression and tending to be clustered within the same genomic neighborhoods

\begin{tabular}{|c|c|c|}
\hline Names & Total & Elements \\
\hline \multirow{11}{*}{$\begin{array}{l}\text { ASXL1 } \\
\text { KIF3B } \\
\text { NOL4L } \\
\text { PLAGL2 } \\
\text { POFUT1 } \\
\text { TM9SF4 }\end{array}$} & \multirow[t]{11}{*}{10} & hsa05213:Endometrial cancer \\
\hline & & hsa04390:Hippo signaling pathway \\
\hline & & hsa04910:Insulin signaling pathway \\
\hline & & hsa 05231 Choline metabolism in cancer \\
\hline & & hsa05200. Pathways in cancer \\
\hline & & 10 \\
\hline & & hsa04919:Thyroid hormone signaling pathway \\
\hline & & hsa04152:AMPK signaling pathway \\
\hline & & hsa04550:Signaling pathways regulating pluripotency of stem cells \\
\hline & & hsa04310:Wnt signaling pathway \\
\hline & & hsa04520:Adherens junction \\
\hline ASXL1 & \multirow[t]{5}{*}{1} & \multirow[t]{5}{*}{ hsa05217:Basal cell carcinoma } \\
\hline KIF3B & & \\
\hline PLAGL2 & & \\
\hline POFUT1 & & \\
\hline TM9SF4 & & \\
\hline ASXL1 & \multirow[t]{5}{*}{5} & hsa04931:Insulin resistance \\
\hline KIF3B & & hsa00310:Lysine degradation \\
\hline $\begin{array}{l}\text { NOL4L } \\
\text { POFUT1 }\end{array}$ & & hsa04012:ErbB signaling pathway \\
\hline \multirow[t]{2}{*}{ TM9SF4 } & & hsa04144:Endocytosis \\
\hline & & hsa05215:Prostate cancer \\
\hline ASXL1 & \multirow[t]{5}{*}{1} & \multirow[t]{5}{*}{ hsa05169:Epstein-Barr virus infection } \\
\hline KIF3B & & \\
\hline NOL4L & & \\
\hline PLAGL2 & & \\
\hline POFUT1 & & \\
\hline KIF3B & \multirow[t]{4}{*}{2} & \multirow{4}{*}{$\begin{array}{c}\text { hsa03320:PPAR signaling pathway } \\
\text { hsa04122:Sulfur relay system }\end{array}$} \\
\hline PLAGL2 & & \\
\hline POFUT1 & & \\
\hline TM9SF4 & & \\
\hline ASXL1 & \multirow[t]{4}{*}{1} & \multirow[t]{4}{*}{ hsa04360:Axon guidance } \\
\hline NOL4L & & \\
\hline PLAGL2 & & \\
\hline TM9SF4 & & \\
\hline KIF3B & \multirow[t]{4}{*}{1} & \multirow[t]{4}{*}{ hsa05219:Bladder cancer } \\
\hline NOL4L & & \\
\hline POFUT1 & & \\
\hline TM9SF4 & & \\
\hline ASXL1 & \multirow[t]{4}{*}{1} & \multirow[t]{4}{*}{ hsa03015:mRNA surveillance pathway } \\
\hline KIF3B & & \\
\hline NOL4L & & \\
\hline POFUT1 & & \\
\hline
\end{tabular}

(Continued) 


\begin{tabular}{|c|c|c|}
\hline Names & Total & Elements \\
\hline \multirow[t]{2}{*}{ NOL4L PLAGL2 TM9SF4 } & \multirow[t]{2}{*}{2} & hsa04015:Rap1 signaling pathway \\
\hline & & hsa04340:Hedgehog signaling pathway \\
\hline KIF3B & \multirow[t]{3}{*}{1} & \multirow[t]{3}{*}{ hsa05210:Colorectal cancer } \\
\hline POFUT1 & & \\
\hline TM9SF4 & & \\
\hline \multirow{3}{*}{$\begin{array}{l}\text { ASXL1 } \\
\text { NOL4L } \\
\text { TM9SF4 }\end{array}$} & \multirow[t]{3}{*}{3} & hsa04530:Tight junction \\
\hline & & hsa04120:Ubiquitin mediated proteolysis \\
\hline & & hsa03018:RNA degradation \\
\hline \multirow{3}{*}{$\begin{array}{l}\text { KIF3B } \\
\text { PLAGL2 } \\
\text { POFUT1 }\end{array}$} & \multirow[t]{3}{*}{3} & hsa04975:Fat digestion and absorption \\
\hline & & hsa03020:RNA polymerase \\
\hline & & hsa04146:Peroxisome \\
\hline \multirow{3}{*}{$\begin{array}{l}\text { ASXL1 } \\
\text { KIF3B } \\
\text { POFUT1 }\end{array}$} & \multirow[t]{3}{*}{3} & hsa03008:Ribosome biogenesis in eukaryotes \\
\hline & & hsa04110:Cell cycle \\
\hline & & hsa03013:RNA transport \\
\hline KIF3B & \multirow[t]{3}{*}{1} & \multirow[t]{3}{*}{ hsa04068:FoxO signaling pathway } \\
\hline NOL4L & & \\
\hline POFUT1 & & \\
\hline \multirow{3}{*}{$\begin{array}{l}\text { PLAGL2 } \\
\text { TM9SF4 }\end{array}$} & \multirow[t]{3}{*}{3} & hsa00561:Glycerolipid metabolism \\
\hline & & hsa04916:Melanogenesis \\
\hline & & hsa04976:Bile secretion \\
\hline \multirow{2}{*}{$\begin{array}{l}\text { ASXL1 } \\
\text { TM9SF4 }\end{array}$} & \multirow[t]{2}{*}{2} & hsa04350:TGF-beta signaling pathway \\
\hline & & hsa04710:Circadian rhythm \\
\hline \multirow{9}{*}{$\begin{array}{l}\text { NOL4L } \\
\text { TM9SF4 }\end{array}$} & \multirow[t]{9}{*}{9} & hsa05223:Non-small cell lung cancer \\
\hline & & hsa04911:Insulin secretion \\
\hline & & hsa04330:Notch signaling pathway \\
\hline & & hsa04666:Fc gamma R-mediated phagocytosis \\
\hline & & hsa05211:Renal cell carcinoma \\
\hline & & hsa04070:Phosphatidylinositol signaling system \\
\hline & & hsa05220:Chronic myeloid leukemia \\
\hline & & hsa05205:Proteoglycans in cancer \\
\hline & & hsa05120:Epithelial cell signaling in Helicobacter pylori infection \\
\hline \multirow{6}{*}{$\begin{array}{l}\text { KIF3B } \\
\text { POFUT1 }\end{array}$} & \multirow[t]{6}{*}{6} & hsa00240:Pyrimidine metabolism \\
\hline & & hsa00072:Synthesis and degradation of ketone bodies \\
\hline & & hsa01100:Metabolic pathways \\
\hline & & hsa00230:Purine metabolism \\
\hline & & hsa00600:Sphingolipid metabolism \\
\hline & & hsa04964:Proximal tubule bicarbonate reclamation \\
\hline
\end{tabular}

The gene-set of each gene (Pearson correlation coefficient $\geq 0.2$, Supplementary Table 8) was compiled from cBioPortal for Cancer Genomics (Colorectal Adenocarcinoma (TCGA [36]) 276 samples). 
and therefore promote pathogenesis and development of colorectal cancer.

\section{DISCUSSION}

The chromosome 20q, as first observed by means of banding analysis in colorectal cancer, frequently exhibits complex copy number amplification patters that have been reported in a variety of other cancer $[2,12]$. Further study thereafter showed gains in $20 \mathrm{q}$ occurs in $>65 \%$ of CRCs, the most significant of which were mostly found in 20q11 and 20q13, Patients in 20q gain were always been associated with poor prognoses [1, 13]. Previous studies have confirmed that CNA plays an important role in the altering expression of genes residing on it. A few example include amplification of the MYC oncogeneof 8 q24 resulting in its over-expression, assuming a role a central driver in CRCs [14], the amplification of 20q, which has been reported causing mRNA overexpression of AURKA (20q13.2) and TPX2 (20q11), ultimately promoting progression from colorectal adenoma to carcinoma [13]. Integrated analysis of copy number variation and genome-wide expression profiling in CRC identified 56 overlapping genes,and 48 of which (85.7\%) showed a positive association [15]. Similar conclusions were obtained in our study including two aspects. For one thing, the top 20 genes with mRNA overexpression in CRC are all located on $20 \mathrm{q}$ and significantly correlated with CNA, which suggest strong cis effects of CNA on mRNA abundance and underlines the important role of $20 \mathrm{q}$ involved in CRCs. For another thing, these up-regulated genes caused by CNA mainly resided on 20q11(20q11.23, $20 q 11.22$ and $20 q 11.21)$ and $20 q 13(20 q 13.33,20 q 13.32$, $20 q 13.31,20 q 13.13$ and $20 q 13.12)$, which is in line with previous studies that show $20 \mathrm{q} 11$ and $20 \mathrm{q} 13$ are the most frequently amplified region.

Even though these genes on $20 \mathrm{q}$ demonstrated mRNA overexpression in great deal of CRCs, the proteins of these didn't show the same result. Certain protein such as PIGU even demonstrated negative immunoreactivity in some CRC tissue, which corresponded to previous studies using integrated proteogenomic analyses that conclude that CNA-mRNA correlations were significantly higher than CNA-protein correlations for genes in all three groups (CNA, mRNA and protein $)(\mathrm{P}<1.0 \times 10-10$, KolmogorovSmirnov test) [8]. All these studies, as well as ours,
$\mathbf{A}$

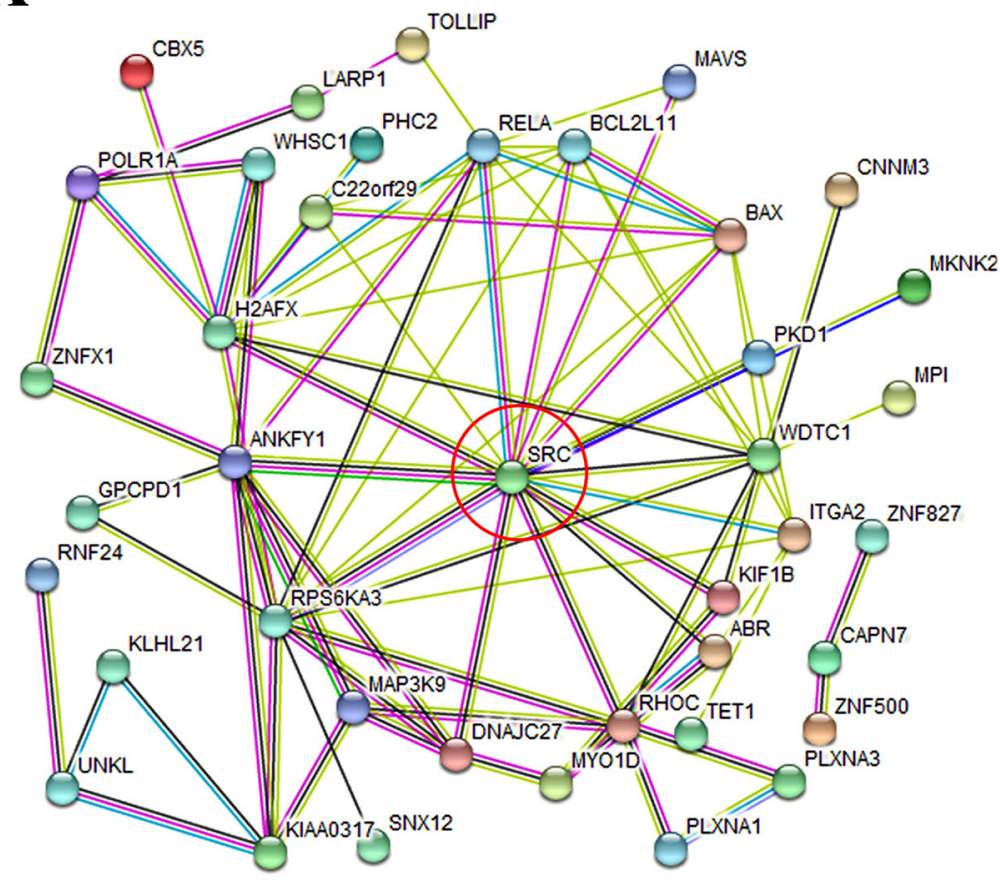

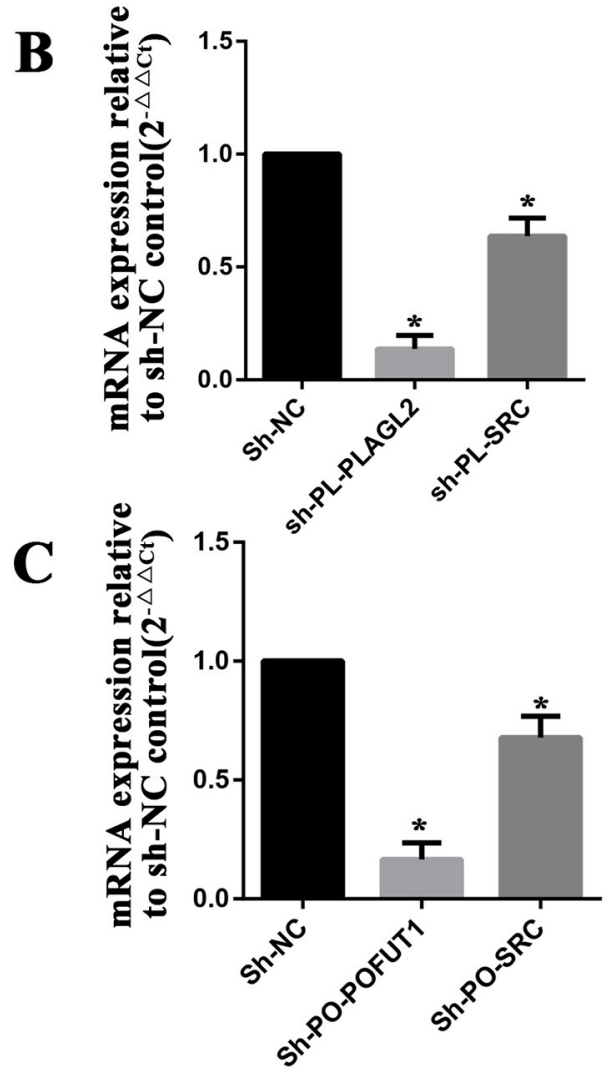

Figure 8: PLAGL2 and POFUT1 cooperatively participated in SRC biological pathways. (A) 63 genes which can maintain correlation with POFUT1 and PLAGL2 in 14 human tumor tissues were selected for protein-protein interactions by STRING v10.5, the oncogenes SRC is the most important intersection point. (B) The knock-out of gene PLAGL2 (sh-PL-PLAGL2) in colorectal cancer cell SW620 led to inhibition of SRC (sh-PL-SRC). (C) The knock-out of gene POFUT1 (sh-PO-POFUT1) in colorectal cancer cell SW620 led to inhibition of SRC (sh-PL-SRC). 
indicated many CNA-driven mRNA level increases do not translate into increased abundance of the corresponding proteins. In addition, we found that 3 genes(TM9SF4, PLAGL2 and POFUT1) with positive CNA-protein and mRNA correlations were not correlated with survival rate of CRC based on the data from the TCGA database. The paradox is that previous research has reported that TM9SF4, a key gene involved in cancer cell cannibalism, is a novel V-ATPase-interacting protein that regulates tumor $\mathrm{pH}$ alterations associated with drug resistance and invasiveness of colon cancer cells [16], and PALGL2, a prognostic factor, can serve as a tumor oncoprotein in the development and progression of colorectal cancer [17]. This highlights the fact that more work needs tobe done with the support of database. It is worth noting that not all genes with CNA can causemRNA over-expression; some even presented negative correlation, such as BCAS1and EDN3 [4, 15].Some researchers recommend that for the research on down-regulated genes in amplified chromosomal areas, which would be are obviously very interesting candidates to evaluate as putative tumor suppressors [18].

Interestingly, we found that these genes with mRNA overexpression showed a strong tendency to co-express, and what's more, these genes with co-expression tend to lie adjacent to each other and formehysically adjacent gene cluster(2-7 genes) in the genome. Similar phenomena have been observed in a variety of species such as Drosophila,mice and humans [9]. Among the potential underlying mechanism, the sharing of regulatory elements such as transcription factors, promoters and enhancers is a key factor $[9,19,20]$. So in our study, an adjacent gene group including TM9SF4, POFUT1, PLAGL2, KIF3B and ASXL1 were subjected to Venn Diagrams analysis and found that they share 60 transcription factors, as supported by ChIP-Seq evidence. The enrichment analysis oftranscription that revealed that adjacent gene pairs and gene triplets are more likely to be modulated by same transcription factors. These results corresponded to previous studies that found that physical distance may play an important role in the co-expression of neighboring genes [19, 20].

As discussed above, these genes with short distance are prime candidates for sharing regulatory elements and presenting co-expression. When they separated of less than $1000 \mathrm{bp}$, one classical example, a bidirectional promoter, can occur. Bidirectional promoters gene may be generated from two genes arranged in a head-to-head(adjacent 5 ' ends) configuration with less than 1000 base pairs of intervening sequence, They are generally evolutionarily conserved, share transcription factors, presente higher GC content and often lack TATA boxes [21]. Expression of gene pairs driven by bidirectional promoters appeares to be correlated, as shown by PSENEN and U2AF1L4 [22], and PRR11 and SKA2 [23]. In our research, PLAGL2 and POFUT1 exhibited significant co-expression levels $(\mathrm{r}=0.84)$ and perfectly met the criteria described above, so we postulated that the co-expression of two genes is regulated by a bidirectional promoter.

It is noteworthy that many adjacent genes are coexpressed, but some including bidirectional pairs are antiregulated [24]. So in this article, we also explored another mechanism, the competitive endogenous RNA (ceRNA) hypothesis, which was recently aroused great interest in studies of human cancer. RNA transcripts that contain miRNA-binding sites can communicate with and indirectly regulate each other by competing specifically for shared miRNAs, in particular, resulting co-expression of RNA transcripts [25]. In our study, we found adjacent gene group, TM9SF4, POFUT1, PLAGL2, KIF3B and ASXL1 can share 9 common predicted miRNAs, especially the significantly correlated gene pair POFUT1 and PLAGL2 share common 52 microRNAs with experimental evidence, and these microRNAs can directly target PLAGL2 and POFUT1 and repress the expression of both genes in colon and rectal cancer, which indicate that ceRNA may be another important mechanism causing co-expression of these adjacent genes.

But the need to pay attention to is that although the co-expression of neighboring genes is a remarkable phenomenon, the mechanism behind that is as yet unclear $[9,19]$. Many mechanisms can explain the co-expression of neighboring genes such as alteration of the chromatin structure, sharing of regulatory elements, chromatin domains, spatial organization and functional relationship of gene clusters $[9,20,26]$. Taking into account the content of the article, we only focused on three main mechanisms in this article. We hold that these mechanisms only can account for the part of the coexpression pattern and may be be able to provide some brief references for further research in this field.

Previous studies have also suggested that genes with co-expression characterization always participate in the same biological function. In our study, 6 adjacent genes with co-expression characterization in Figure 3(TM9SF4, PLAGL2, POFUT1, KIF3B, ASXL1, NOL4L) were chosen for gene-set pathway enrichment analysis. We found that these overexpressed adjacent genes caused by CNA in colorectal cancer can cooperatively participate in the same biological pathways associated with human cancers, including colorectal cancer.Additionally, we inhibited the expression of PLAGL2 and POFUT1 in SW620 cells by shRNA, the result found that PLAGL2 and POFUT1 both can be involved in $S R C$ biological pathways. Recent studies have confirmed that several of the 6 genes discussed above are involved in human cancer, especially colorectal cancer., TM9SF4 is a novel $\mathrm{V}$-ATPase-interacting protein that modulates tumor $\mathrm{pH}$ alterations associated with drug resistance and invasiveness of colon cancer cells [16], PLAGL2 induces epithelial-mesenchymal transition via $\mathrm{Wnt} / \beta$-catenin signaling pathway in human colorectal adenocarcinoma 
[27] and overexpression of protein POUT1 accelerates hepatocellular carcinoma progression via the Notch signaling pathway [28]. It's remarkable that the function of V-ATPases in cancer was closely related to the wnt and notch signaling pathway [29], which indicateds to us that PLAGL2, POFUT1 and TM9SF4 may cooperatively participated in the development human cancer. In addition, KIF3B and ASXL1 alsohave the potential to participatein human cancer $[30,31]$.

In general, in this study we firstly identified that most frequently unregulated genes caused by CNA in colorectal cancer are all located on chromosome 20q.Upon investigation, we found that these genes demonstrated coexpression features and tended to be clustered within the same genomic neighborhoods, and then tried to explore the underlying mechanism by looking into the sharing of transcription factors, conserved bidirectional promoter and ceRNA hypotheses. But whether these mechanisms are synergistic or independent, more work needs to be done to better understand how these adjacent genes of $20 \mathrm{q}$ maintain co-expression in colorectal cancer. In addition, we found that co-expression of adjacent genes which cooperatively participatein the same biological pathways can be associated with the pathogenesis and development of human cancer, especially colorectal cancer; This means that there are now novel genomic targets for therapeutic intervention for colorectal cancer.

\section{MATERIALS AND METHODS}

\section{The information and chromosomal distribution of genes located on 20q}

The official symbol, official full name, location, cytoband and gene type of all genes on chromosome 20 conformed to human species assembly version GRCh38(NCBI and Ensemble). The gene organization, $\mathrm{CpG}$ islands, chromatin state annotation, transcriptional regulation and evolutionary conservation were visualized by tracks through UCSC Genome Browser(http://genome. ucsc.edu/). The data for adjacent gene analysis was downloaded from NCBI Map Viewer (https://www.ncbi. nlm.nih.gov/projects/mapview).(more details can be found in Supplementary Table 2).

\section{Somatic mutations and expression of genes}

COSMIC (the Catalogue Of Somatic Mutations In Cancer) study on colon adenocarcinoma (Study Id COSU376)[32], cBioPortal [33] for Cancer Genomics (Colorectal Adenocarcinoma (TCGA, Provisional) 633 samples and Colorectal Adenocarcinoma (TCGA, Nature 2012) 276 samples) and Oncomine(GSE9348 and TCGA Colorectal) were used for somatic mutations and expression analysis of all genes [34].

\section{Protein expression and survival analysis}

The protein expression of all genes were evaluated by The Human Protein Atlas project [35] and the expression data is derived from antibodybased protein profiling using immunohistochemistry. Survival analysis was performed by OncoLnc, a database that can link TCGA survival data to mRNA, miRNA, or lncRNA expression levels (http://www. oncolnc.org/).

\section{Evaluating CNA-mRNA, miRNA-mRNA, mRNA-mRNA and mRNA (transcription factor)-mRNA correlation}

We downloaded mRNA and CNV data for colon and rectal cancer from the Cancer Genome Atlas(TCGA) portal [36],.Copy-number alterations and gene-expression data for all 276 samples were detected by Affymetrix SNP 6.0 microarrays, Illumina HiSeq, Agilent microarrays and RNA-Seq. 218 data setswith quantifiable CNA and mRNA measurements from TCGA(Supplementary Table 1) were used for CNA-mRNA evaluation and mRNAmRNA evaluation of 3 genes (PLAGL2, POFUT1 and STAU1). 244 data sets (Supplementary Table 4) were used for mRNA (transcription factor)-mRNA correlation evaluation. starBase v2.0 [37], which provide miRNAtarget interactions processed from five miRNA prediction software programs and overlapped with CLIP-Seq data, was used to explore anti-correlation (pearson correlation: $\mathrm{r}<0$, p-value $<0.05$ ) between miRNA and target genes across colon and rectal cancer. Additionally, relationships between genes were analyzed by GeneMANIA [38], a free public resource that offers a simple, intuitive web interface.

\section{Identification of regulatory transcription factors within promoters}

Prediction of putative transcription factor binding sites was performed using software GeneCard [39] and the JASPAR database [40]. Gene Card provided a link to the Ensembl regulatory element, promoter length and a list of TFs (Transcription Factors) having TFBSs (Transcription Factor Binding Sites) within the promoter (based on ChIP-Seq evidence). Comprehensive gene set enrichment analysis for transcriptional machinery was carried out using the software Enrichr [41]. 2 libraries including position weight matrices (PWMs) from TRANSFAC and JASPAR, and transcription factor targets extracted from the Encyclopedia of DNA Elements(ENCODE) project were selected to identify transcription factors that are enriched for target genes within the input list. 


\section{miRNA-mRNA intersection and ceRNA identification}

TargetScan 7.1, which has been proven to have with higher sensitivity and precision than other target predicting programs, was used to predict biological miRNA-mRNA intersection [42]. The experimentally validated microRNAs were compiled from TarBase v7.0 [43] and miRTARBASE 6.0 [44], the predicted miRNA-target interactions were processed from five miRNA prediction software programs (TargetScan, PicTar, PITA, miRanda and RNA22) and overlapped with CLIP-Seq data compiled from Starbase v2.0; In addition, Starbase v2.0 was employed to predict competing endogenous RNA (ceRNA) pairs by integrating the interactions from potential microRNA targets (miRanda/mirSVR) which overlapped with CLIP-Seq data [37], and to select the genes which can maintain correlation with POFUT1 and PLAGL2 in 14 human tumor tissues.

\section{Analysis of biological networks}

All enrichment analyses of KEGG pathways were identified statistically with DAVID Bioinformatics Resources 6.8 [45],.STRING v10.5 [46] was used to provide a critical assessment and integration of proteinprotein interactions.

\section{RT-qPCR}

Total RNA was extracted by Trizol (Invitrogen) and reverse transcribed using ReverTra Ace qPCR RT Master Mix with gDNA Remover (TOYOBO).qPCR assays were performed by using KOD SYBR $®$ qPCR Mix (TOYOBO) on LightCycler ${ }^{\circledR}$ 480II System (Roche) according to the manufacturer's instructions. Treated samples were normalized to controls with the ${ }^{\Delta \Lambda} \mathrm{Ct}$ formula using GAPDH as an endogenous control. The primer used in this study can be found in Supplementary Table 6 .

\section{Cell culture and transfection}

The human colorectal cancer cell SW620 was maintained in L-15 medium supplemented with $10 \%$ fetal bovine serum, penicillin and streptomycin, and incubated at $37^{\circ} \mathrm{C}$ in $5 \% \mathrm{CO}$. All Lentivirus shRNA were synthesized by Suzhou GenePharma(Suzhou, China). The shRNA of PLAGL2 and POFUT1 were transfected into the SW620 according to the manufacturer's instructions. Cells were then collected and subjected to analysis after showing stability.

\section{Statistical analyses}

Linear regression and correlation studies were performed using the software GraphPad Prism 7 (San
Diego, California, USA). Linear regression finds the best line that predicts $\mathrm{Y}$ from $\mathrm{X}$. Correlation computes the value of the Pearson correlation coefficient, $r$, and its value ranges from -1 to +1 . All correlation analysis, including CNA-mRNA, mRNA-mRNA and mRNA (transcription factor)-mRNA, results (mean values $\pm \mathrm{SD}$ ) were subjected to statistical analysis by $t$-test, in which $\mathrm{p}<0.05$ was considered statistically significant. The software Venn Diagrams (http://bioinformatics.psb. ugent.be/) was used for calculating the intersection(s) of list of elements.

\section{ACKNOWLEDGMENTS}

This work was supported by National Nature Science Foundation of China (No.81602568); The New Xiangya Talent Projects of the Third Xiangya Hospital of Central South University (No.20150306, No.JY201611). We express our great appreciation to Arad jain to modify and improve our paper, especially the language modification.

\section{CONFLICTS OF INTEREST}

The authors declare that they have no competing interest.

\section{REFERENCES}

1. Carvalho B, Postma C, Mongera S, Hopmans E, Diskin $\mathrm{S}$, van de Wiel MA, van Criekinge W, Thas O, Matthai A, Cuesta MA, Terhaar Sive Droste JS, Craanen M, Schrock E, et al. Multiple putative oncogenes at the chromosome 20q amplicon contribute to colorectal adenoma to carcinoma progression. Gut. 2009; 58: 79-89. https://doi.org/10.1136/ gut.2007.143065.

2. Muleris M, Salmon RJ, Dutrillaux AM, Vielh P, Zafrani B, Girodet J, Dutrillaux B. Characteristic chromosomal imbalances in 18 near-diploid colorectal tumors. Cancer Genet Cytogenet. 1987; 29: 289-301.

3. De Angelis PM, Clausen OP, Schjolberg A, Stokke T. Chromosomal gains and losses in primary colorectal carcinomas detected by $\mathrm{CGH}$ and their associations with tumour DNA ploidy, genotypes and phenotypes. Br J Cancer. 1999; 80: 526-35. https://doi.org/10.1038/ sj.bjc.6690388.

4. Platzer P, Upender MB, Wilson K, Willis J, Lutterbaugh J, Nosrati A, Willson JK, Mack D, Ried T, Markowitz S. Silence of chromosomal amplifications in colon cancer. Cancer Res. 2002; 62: 1134-8.

5. Wang Z, Liu Y, Zhang P, Zhang W, Wang W, Curr K, Wei G, Mao JH. FAM83D promotes cell proliferation and motility by downregulating tumor suppressor gene FBXW7. Oncotarget. 2013; 4: 2476-86. https://doi.org/10.18632/ oncotarget.1581. 
6. Fortney K, Jurisica I. Integrative computational biology for cancer research. Hum Genet. 2011; 130: 465-81. https://doi. org/10.1007/s00439-011-0983-z.

7. Weber GW, Ozogur-Akyuz S, Kropat E. A review on data mining and continuous optimization applications in computational biology and medicine. Birth Defects Res C Embryo Today. 2009; 87: 165-81. https://doi.org/10.1002/ bdrc. 20151.

8. Zhang B, Wang J, Wang X, Zhu J, Liu Q, Shi Z, Chambers MC, Zimmerman LJ, Shaddox KF, Kim S, Davies SR, Wang S, Wang P, et al. Proteogenomic characterization of human colon and rectal cancer. Nature. 2014; 513: 382-7. https://doi.org/10.1038/nature13438.

9. Michalak P. Coexpression, coregulation, and cofunctionality of neighboring genes in eukaryotic genomes. Genomics. 2008; 91: 243-8. https://doi.org/10.1016/j.ygeno.2007.11.002.

10. Yang MQ, Koehly LM, Elnitski LL. Comprehensive annotation of bidirectional promoters identifies co-regulation among breast and ovarian cancer genes. PLoS Comput Biol. 2007; 3: e72. https://doi.org/10.1371/journal. pcbi.0030072.

11. Salmena L, Poliseno L, Tay Y, Kats L, Pandolfi PP. A ceRNA hypothesis: the Rosetta Stone of a hidden RNA language? Cell. 2011; 146: 353-8. https://doi.org/10.1016/j. cell.2011.07.014.

12. Hodgson JG, Chin K, Collins C, Gray JW. Genome amplification of chromosome 20 in breast cancer. Breast Cancer Res Treat. 2003; 78: 337-45.

13. Sillars-Hardebol AH, Carvalho B, Tijssen M, Belien JA, de Wit M, Delis-van Diemen PM, Ponten F, van de Wiel MA, Fijneman RJ, Meijer GA. TPX2 and AURKA promote $20 \mathrm{q}$ amplicon-driven colorectal adenoma to carcinoma progression. Gut. 2012; 61: 1568-75. https://doi. org/10.1136/gutjnl-2011-301153.

14. Camps J, Nguyen QT, Padilla-Nash HM, Knutsen T, McNeil NE, Wangsa D, Hummon AB, Grade M, Ried $\mathrm{T}$, Difilippantonio MJ. Integrative genomics reveals mechanisms of copy number alterations responsible for transcriptional deregulation in colorectal cancer. Genes Chromosomes Cancer. 2009; 48: 1002-17. https://doi. org/10.1002/gcc.20699.

15. Ali Hassan NZ, Mokhtar NM, Kok Sin T, Mohamed Rose I, Sagap I, Harun R, Jamal R. Integrated analysis of copy number variation and genome-wide expression profiling in colorectal cancer tissues. PLoS One. 2014; 9: e92553. https://doi.org/10.1371/journal.pone.0092553.

16. Lozupone F, Borghi M, Marzoli F, Azzarito T, Matarrese P, Iessi E, Venturi G, Meschini S, Canitano A, Bona R, Cara A, Fais S. TM9SF4 is a novel V-ATPase-interacting protein that modulates tumor $\mathrm{pH}$ alterations associated with drug resistance and invasiveness of colon cancer cells. Oncogene. 2015; 34: 5163-74. https://doi.org/10.1038/ onc.2014.437.
17. Liu B, Lu C, Song YX, Gao P, Sun JX, Chen XW, Wang MX, Dong YL, Xu HM, Wang ZN. The role of pleomorphic adenoma gene-like 2 in gastrointestinal cancer development, progression, and prognosis. Int J Clin Exp Pathol. 2014; 7: 3089-100.

18. Kotliarov Y, Kotliarova S, Charong N, Li A, Walling J, Aquilanti E, Ahn S, Steed ME, Su Q, Center A, Zenklusen JC, Fine HA. Correlation analysis between singlenucleotide polymorphism and expression arrays in gliomas identifies potentially relevant target genes. Cancer Res. 2009; 69: 1596-603. https://doi.org/10.1158/0008-5472. can-08-2496.

19. Tsai HK, Huang PY, Kao CY, Wang D. Co-expression of neighboring genes in the zebrafish (Danio rerio) genome. Int J Mol Sci. 2009; 10: 3658-70. https://doi.org/10.3390/ ijms 10083658 .

20. Vogel JH, von Heydebreck A, Purmann A, Sperling S. Chromosomal clustering of a human transcriptome reveals regulatory background. BMC Bioinformatics. 2005; 6: 230. https://doi.org/10.1186/1471-2105-6-230.

21. Wakano C, Byun JS, Di LJ, Gardner K. The dual lives of bidirectional promoters. Biochim Biophys Acta. 2012; 1819: 688-93. https://doi.org/10.1016/j.bbagrm.2012.02.006.

22. Didych DA, Shamsutdinov MF, Smirnov NA, Akopov SB, Monastyrskaya GS, Uspenskaya NY, Nikolaev LG, Sverdlov ED. Human PSENEN and U2AF1L4 genes are concertedly regulated by a genuine bidirectional promoter. Gene. 2013; 515: 34-41. https://doi.org/10.1016/j. gene.2012.11.058.

23. Wang Y, Zhang Y, Zhang C, Weng H, Li Y, Cai W, Xie M, Long Y, Ai Q, Liu Z, Du G, Wang S, Niu Y, et al. The gene pair PRR11 and SKA2 shares a NF-Y-regulated bidirectional promoter and contributes to lung cancer development. Biochim Biophys Acta. 2015; 1849: 1133-44. https://doi.org/10.1016/j.bbagrm.2015.07.002.

24. Trinklein ND, Aldred SF, Hartman SJ, Schroeder DI, Otillar RP, Myers RM. An abundance of bidirectional promoters in the human genome. Genome Res. 2004; 14: 62-6. https:// doi.org/10.1101/gr.1982804.

25. Tay Y, Rinn J, Pandolfi PP. The multilayered complexity of ceRNA crosstalk and competition. Nature. 2014; 505: 34452. https://doi.org/10.1038/nature12986.

26. Rieder D, Ploner C, Krogsdam AM, Stocker G, Fischer M, Scheideler M, Dani C, Amri EZ, Muller WG, McNally JG, Trajanoski Z. Co-expressed genes prepositioned in spatial neighborhoods stochastically associate with SC35 speckles and RNA polymerase II factories. Cell Mol Life Sci. 2014; 71: 1741-59. https://doi.org/10.1007/s00018-013-1465-3.

27. Wang YP, Guo PT, Zhu Z, Zhang H, Xu Y, Chen YZ, Liu F, Ma SP. Pleomorphic adenoma gene like-2 induces epithelialmesenchymal transition via Wnt/beta-catenin signaling pathway in human colorectal adenocarcinoma. Oncol Rep. 2017; 37: 1961-70. https://doi.org/10.3892/or.2017.5485. 
28. Ma L, Dong P, Liu L, Gao Q, Duan M, Zhang S, Chen S, Xue R, Wang X. Overexpression of protein O-fucosyltransferase 1 accelerates hepatocellular carcinoma progression via the Notch signaling pathway. Biochem Biophys Res Commun. 2016; 473: 503-10. https://doi. org/10.1016/j.bbrc.2016.03.062.

29. Stransky L, Cotter K, Forgac M. The function of V-ATPases in cancer. Physiol Rev. 2016; 96: 1071-91. https://doi. org/10.1152/physrev.00035.2015.

30. Katoh M. Functional and cancer genomics of ASXL family members. Br J Cancer. 2013; 109: 299-306. https://doi. org/10.1038/bjc.2013.281.

31. Scotto L, Narayan G, Nandula SV, Arias-Pulido H, Subramaniyam S, Schneider A, Kaufmann AM, Wright JD, Pothuri B, Mansukhani M, Murty VV. Identification of copy number gain and overexpressed genes on chromosome arm 20q by an integrative genomic approach in cervical cancer: potential role in progression. Genes Chromosomes Cancer. 2008; 47: 755-65. https://doi.org/10.1002/gcc.20577.

32. Forbes SA, Beare D, Boutselakis H, Bamford S, Bindal N, Tate J, Cole CG, Ward S, Dawson E, Ponting L, Stefancsik R, Harsha B, Kok CY, et al. COSMIC: somatic cancer genetics at high-resolution. Nucleic Acids Res. 2017; 45: D777-83. https://doi.org/10.1093/nar/gkw1121.

33. Cerami E, Gao J, Dogrusoz U, Gross BE, Sumer SO, Aksoy BA, Jacobsen A, Byrne CJ, Heuer ML, Larsson E, Antipin Y, Reva B, Goldberg AP, et al. The cBio cancer genomics portal: an open platform for exploring multidimensional cancer genomics data. Cancer Discov. 2012; 2: 401-4. https://doi.org/10.1158/2159-8290.cd-12-0095.

34. Rhodes DR, Yu J, Shanker K, Deshpande N, Varambally R, Ghosh D, Barrette T, Pandey A, Chinnaiyan AM. ONCOMINE: a cancer microarray database and integrated data-mining platform. Neoplasia. 2004; 6: 1-6.

35. Uhlen M, Fagerberg L, Hallstrom BM, Lindskog C, Oksvold P, Mardinoglu A, Sivertsson A, Kampf C, Sjostedt E, Asplund A, Olsson I, Edlund K, Lundberg E, et al. Proteomics. Tissuebased map of the human proteome. Science. 2015; 347: 1260419. https://doi.org/10.1126/science.1260419.

36. Cancer Genome Atlas Network. Comprehensive molecular characterization of human colon and rectal cancer. Nature. 2012; 487: 330-7. https://doi.org/10.1038/nature11252.

37. Li JH, Liu S, Zhou H, Qu LH, Yang JH. starBase v2.0: decoding miRNA-ceRNA, miRNA-ncRNA and proteinRNA interaction networks from large-scale CLIP-Seq data. Nucleic Acids Res. 2014; 42: D92-7. https://doi. org/10.1093/nar/gkt1248.

38. Montojo J, Zuberi K, Rodriguez H, Kazi F, Wright G, Donaldson SL, Morris Q, Bader GD. GeneMANIA
Cytoscape plugin: fast gene function predictions on the desktop. Bioinformatics. 2010; 26: 2927-8. https://doi. org/10.1093/bioinformatics/btq562.

39. Safran M, Dalah I, Alexander J, Rosen N, Iny Stein T, Shmoish M, Nativ N, Bahir I, Doniger T, Krug H, SirotaMadi A, Olender T, Golan Y, et al. GeneCards Version 3: the human gene integrator. Database (Oxford). 2010; 2010: baq020. https://doi.org/10.1093/database/baq020.

40. Mathelier A, Fornes O, Arenillas DJ, Chen CY, Denay G, Lee J, Shi W, Shyr C, Tan G, Worsley-Hunt R, Zhang AW, Parcy F, Lenhard B, et al. JASPAR 2016: a major expansion and update of the open-access database of transcription factor binding profiles. Nucleic Acids Res. 2016; 44: D1105. https://doi.org/10.1093/nar/gkv1176.

41. Kuleshov MV, Jones MR, Rouillard AD, Fernandez NF, Duan Q, Wang Z, Koplev S, Jenkins SL, Jagodnik KM, Lachmann A, McDermott MG, Monteiro CD, Gundersen GW, et al. Enrichr: a comprehensive gene set enrichment analysis web server 2016 update. Nucleic Acids Res. 2016; 44: W90-7. https://doi.org/10.1093/nar/gkw377.

42. Alexiou P, Maragkakis M, Papadopoulos GL, Reczko M, Hatzigeorgiou AG. Lost in translation: an assessment and perspective for computational microRNA target identification. Bioinformatics. 2009; 25: 3049-55. https:// doi.org/10.1093/bioinformatics/btp565.

43. Vlachos IS, Paraskevopoulou MD, Karagkouni D, Georgakilas G, Vergoulis T, Kanellos I, Anastasopoulos IL, Maniou S, Karathanou K, Kalfakakou D, Fevgas A, Dalamagas T, Hatzigeorgiou AG. DIANA-TarBase v7.0: indexing more than half a million experimentally supported miRNA:mRNA interactions. Nucleic Acids Res. 2015; 43: D153-9. https://doi.org/10.1093/nar/gku1215.

44. Chou CH, Chang NW, Shrestha S, Hsu SD, Lin YL, Lee WH, Yang CD, Hong HC, Wei TY, Tu SJ, Tsai TR, Ho SY, Jian TY, et al. miRTarBase 2016: updates to the experimentally validated miRNA-target interactions database. Nucleic Acids Res. 2016; 44: D239-47. https:// doi.org/10.1093/nar/gkv1258.

45. Huang da W, Sherman BT, Lempicki RA. Systematic and integrative analysis of large gene lists using DAVID bioinformatics resources. Nat Protoc. 2009; 4: 44-57. https://doi.org/10.1038/nprot.2008.211.

46. Szklarczyk D, Franceschini A, Wyder S, Forslund K, Heller D, Huerta-Cepas J, Simonovic M, Roth A, Santos A, Tsafou KP, Kuhn M, Bork P, Jensen LJ, et al. STRING v10: protein-protein interaction networks, integrated over the tree of life. Nucleic Acids Res. 2015; 43: D447-52. https:// doi.org/10.1093/nar/gku1003. 